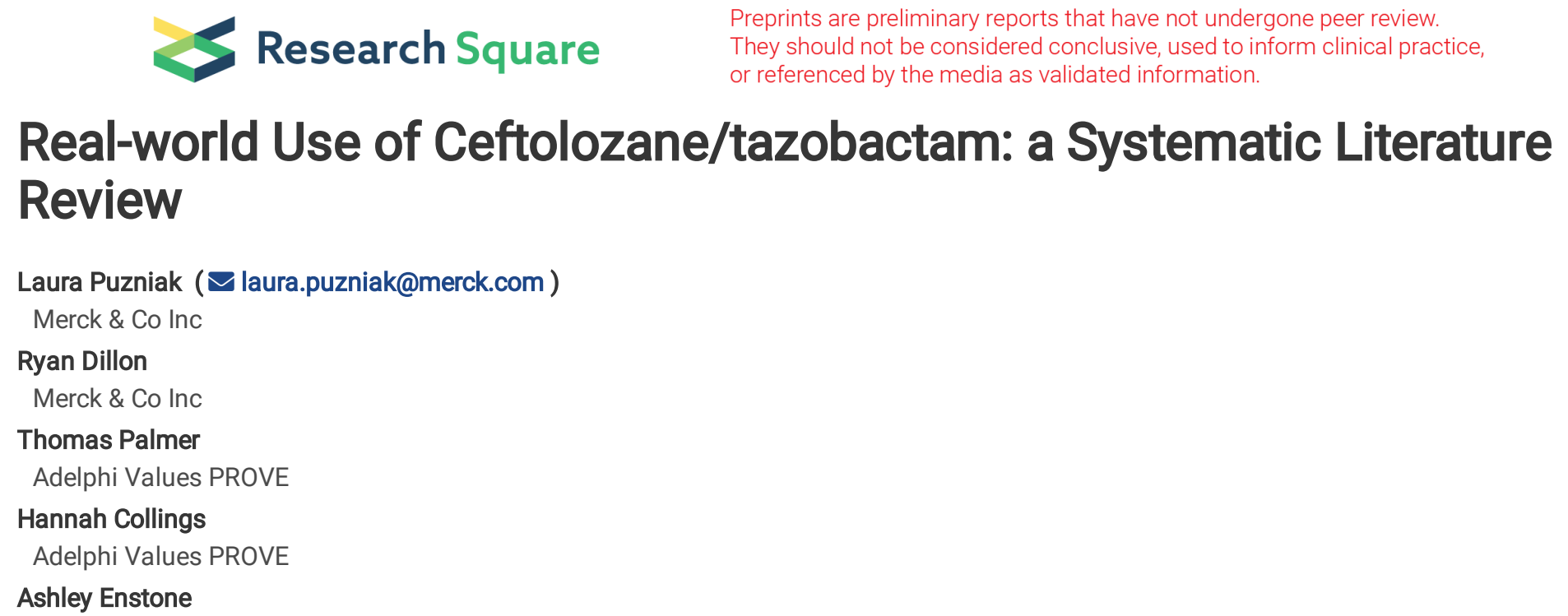

Preprints are preliminary reports that have not undergone peer review.

They should not be considered conclusive, used to inform clinical practice, or referenced by the media as validated information.

\section{Real-world Use of Ceftolozane/tazobactam: a Systematic Literature Review}

\title{
Research
}

Keywords: Ceftolozane/tazobactam, Pseudomonas aeruginosa, antibacterial resistance, real-world evidence

Posted Date: December 30th, 2020

DOI: https://doi.org/10.21203/rs.3.rs-136462/v1

License: (c) (i) This work is licensed under a Creative Commons Attribution 4.0 International License. Read Full License

Version of Record: A version of this preprint was published at Antimicrobial Resistance and Infection Control on April 8th, 2021. See the published version at https://doi.org/10.1186/s13756-021-00933-8. 


\section{Abstract}

Background: Antibacterial-resistant gram-negative infections are a serious risk to global public health. Resistant Enterobacterales and Pseudomonas aeruginosa are highly prevalent, particularly in healthcare settings, and there are limited effective treatment options. Patients with infections caused by resistant pathogens have considerably worse outcomes, and significantly higher costs, relative to patients with susceptible infections. Ceftolozane/tazobactam (C/T) has established efficacy in clinical trials. This review aimed to collate data on $\mathrm{C} / \mathrm{T}$ use in clinical practice.

Methods: This systematic literature review searched online biomedical databases for real-world studies of C/T for gram-negative infections. Relevant study, patient, and treatment characteristics, microbiology, and efficacy outcomes were captured.

Results: There were 83 studies comprising 3,701 patients were identified. The most common infections were respiratory infections ( $52.9 \%$ of reported infections), urinary tract infections (UTIs; $14.9 \%$ ), and intra-abdominal infections (IAls; $10.1 \%)$. Most patients included were seriously ill and had multiple comorbidities. The majority of patients had infections caused by $P$. aeruginosa $(90.7 \%)$, of which $86.0 \%$ were antimicrobial-resistant. C/T was used as both a $1.5 \mathrm{~g}$ q8h and $3 \mathrm{~g} \mathrm{q} 8 \mathrm{~h}$ dose, for a median duration of 7-56 days (varying between studies). Outcome rates were comparable between studies: clinical success rates ranged from 45.7-100.0\%, with 27 studies (69\%) reporting clinical success rates of $>70 \%$; microbiological success rates ranged from $31-100 \%$, with 14 studies $(74 \%)$ reporting microbiological success rates of $>70 \%$. Mortality rates ranged from $0-50 \%$, with 31 studies (69\%) reporting mortality rates of $\leq 20 \%$. In comparative studies, $C / T$ was as effective as aminoglycoside- or polymyxin-based regimens, and in some instances, significantly more effective.

Conclusions: The studies identified in this review demonstrate that $\mathrm{C} / \mathrm{T}$ is effective in clinical practice, despite the diverse group of seriously ill patients, different levels of resistance of the pathogens treated, and varying dosing regimens used. Furthermore, comparative studies suggest that $\mathrm{C} / \mathrm{T}$ offers a successful alternative to standard of care (SoC).

\section{Background}

Antibacterial resistance is a serious risk to global public health. The problem of resistance is especially acute for gram-negative pathogens. (1) Enterobacterales and Pseudomonas aeruginosa are the most prevalent gram-negative hospital-acquired infections (HAls), collectively accounting for $30 \%$ of all HAls in the United States (US).(2) Patients in intensive care units (ICUs) are particularly vulnerable to gramnegative infections and accounts for $70 \%$ of the HAls acquired in ICUs.(2-4)

The burden of infections caused by these pathogens are intensified because of limited effective treatment options. Pathogen susceptibility to many of the available gram-negative antibacterial agents have diminished over time.(5) Patients with infections caused by resistant pathogens have considerably worse outcomes relative to their susceptible counterparts. $(6,7)$ In a US national database study, patients with multidrug-resistant (MDR) P. aeruginosa respiratory infections had higher mortality, an approximately 7-day longer length of stay (LOS), $\$ 20,000$ excess costs, higher readmission rates, and $>\$ 10,000$ excess net loss per case for the hospital relative to those with non-MDR $P$. aeruginosa infections.(7) Further, when the infection is caused by resistant pathogens, it increases the likelihood for receipt of initial inappropriate antibacterial therapy, which has been shown to diminish clinical outcomes and increase costs. $(8,9)$

The challenge of resistance and deleterious impact on outcomes is further compounded by the serious drug-related toxicity associated with some of the current treatment options for resistant gram-negative pathogens. Aminoglycosides (e.g. gentamicin, tobramycin and amikacin) and polymyxins (e.g. colistin) are reported to cause nephrotoxicity and/or ototoxicity. $(10,11)$ Although these antibacterial agents tend to have higher susceptibility to many gram-negative pathogen, they come at a cost of toxicity.

Due to this imminent threat of drug-resistant Enterobacterales and $P$. aeruginosa, and the limited treatment options and toxic effects of some antibacterial agents, the World Health Organization (WHO) in 2017 designated both Enterobacterales and $P$. aeruginosa as the highest 'critical' priority in need of new therapies to counteract this crisis.(12)

Ceftolozane/tazobactam (C/T) is a $\beta$-lactam/ $\beta$-lactamase inhibitor antibacterial agent, consisting of a fixed (2:1) combination of an antipseudomonal cephalosporin, ceftolozane, and the well-established $\beta$-lactamase inhibitor, tazobactam.(13) $\mathrm{C} / \mathrm{T}$ is approved in the US and Europe for clinical use in adults with complicated urinary tract infections (cUTIs), including pyelonephritis, complicated intra-abdominal infections (clAls), and hospital-acquired bacterial pneumonia/ventilator-associated bacterial pneumonia (HABP/VABP). $(13,14)$ The approval of $\mathrm{C} / \mathrm{T}$ was supported by three multinational, randomized, double-blind, active comparator-controlled trials: ASPECT-cUTI, ASPECTCIAl and ASEPCT-NP.(15-17) In the ASPECT trials, C/T demonstrated superiority over levofloxacin (ASPECT-cUTI), and noninferiority to meropenem (ASPECT-cIAl and -NP).(15-17) Since launch in 2014, real-world evidence (RWE) for the use of C/T in clinical practice has been 
accumulating. The purpose of this systematic literature review (SLR) was to identify and collate published RWE to better understand the characteristics of patients treated with $\mathrm{C} / \mathrm{T}$ and clinical outcomes.

\section{Methodology}

\subsection{Literature search}

A search of the literature for C/T RWE, published between 1st January 2009 and 3rd June 2020, was conducted in the following biomedical and economic databases via the OVID platform: Embase, MEDLINE, Psyclnfo, Econlit, and EBM Reviews (ACP Journal Club, Cochrane Database of Systematic Reviews, Cochrane Methodology Register, Database of Abstracts of Reviews of Effects, Health Technology Assessment, NHS Economic Evaluation Database, Cochrane Clinical Answers). The search was conducted in January 2019 with a 10-year time horizon, then re-ran to capture literature published between January-November 2019, and November 2019-June 2020. The time horizon was chosen to minimize erroneous data identification given that C/T was approved for use in 2014 - using a longer horizon would capture any publications reporting on expanded access or compassionate use. The search was limited to English Language publications only.

Due to the heterogeneity of reporting of RWE, the search was designed to be broad to ensure relevant studies which may not be appropriately indexed were retrieved. Table 1 details the search strategy.

Table 1

OVID search strategy

\begin{tabular}{|ll|}
\hline$\#$ & Search terms \\
\hline 1 & Ceftolozane/ OR Ceftolozane plus tazobactam/ \\
\hline 2 & ((Ceftolozane adj1 tazobactam) OR ZERBAXA OR MK-7625A).ti,ab. \\
\hline 3 & 1 OR 2 \\
\hline 4 & (exp animals/ OR nonhuman/) NOT exp human/ \\
\hline 5 & exp controlled clinical trial/ \\
\hline 6 & 4 OR 5 \\
\hline 7 & 3 NOT 6 \\
\hline TOTAL (deduplicated and limits* applied) \\
\hline *English and 2009-current. \\
\hline
\end{tabular}

A further search of internet-based sources relating to C/T RWE was also conducted (limited to English language only). This gray literature review involved searching conference proceedings of two conferences (European Congress of Clinical Microbiology and Infectious Diseases [ECCMID] and Infectious Disease Week [IDWeek]) - two of the largest infectious disease conferences in Europe and the US. Conference proceedings, when published as part of an abstract book, were also identified during the OVID search.

\subsection{Study selection}

All screening (by title and abstract, and by full-text) was performed by two reviewers and any uncertainties were resolved by a third reviewer. Predetermined inclusion and exclusion criteria were used to assess the eligibility of identified abstracts and full-texts for inclusion. PICOS eligibility criteria included observational and non-controlled studies reporting on the use of $\mathrm{C} / \mathrm{T}$ to treat adult patients ( $\geq 18$ years of age) with gram-negative infections in real-world clinical practice. Only studies in English were included. Studies were excluded if they did not meet the PICOS criteria, such as randomized controlled trials (RCTs) or other randomized or controlled experimental studies (Table S1; supplementary material).

\subsection{Data extraction and analysis}

Relevant study, patient, and treatment characteristics, microbiology, and efficacy outcomes were extracted into a data extraction form by one reviewer and checked by a senior reviewer. Efficacy outcomes included clinical cure (typically defined as the resolution of signs or symptoms 
of infection following therapy and survival), microbiological cure (typically defined as large reduction or eradication in the number of pathogens following therapy), and mortality.

\section{Results}

\subsection{SLR results}

A total of 1,222 records were identified from the database searches, and 23 records were identified from the gray literature search. This resulted in 874 non-duplicate records that were subject to title and abstract screening. A total of 730 records were excluded according to the PICOS criteria and 144 were included for full-text review. Of these, 83 studies were determined to be eligible for data extraction and qualitative synthesis. The results of the SLR and study selection processes are presented in Fig. 1.

\subsection{Study characteristics}

Of the 83 studies included in the SLR, 61 were published as peer-reviewed publications,(18-78) and 22 were conference proceedings (availability as abstracts or posters).(79-100) Including studies that recruited patients from multiple countries, the most common study locations were the US $(N=50),(21,22,24,27-29,33,34,39-41,43-45,50,51,54,56,57,59,61,62,68-71,73-77,79-85,87-92,94,95$, 97-100) Spain $(\mathrm{N}=15),(26,28,30-32,35-37,42,47,49,58,66,79,96)$ and Italy $(\mathrm{N}=13) .(18,20,23,25,48,52,53,55,64,67,72,79,86) \mathrm{A}$ variety of study designs were captured: 27 were non-comparative retrospective studies, $(18,19,22,24,25,28,32,33,40,41,79,81,84-92$, 94-99) 14 were case series, $(20,21,29,31,34-39,42,43,82,100)$ five were comparative (including two cohort studies,(80, 83) and three case-control studies, $(23,26,27))$ and one was a non-comparative prospective study. $(30)$ There were thirty-six single-patient case reports identified. $(44-78,93)$ Case reports were included to capture uses of $C / T$ in special clinical situations. Table S2 in the supplementary material summarizes the single-patient case reports identified by the SLR. There were 47 studies (24 multicenter) reporting on more than one patient, as summarized in Table 2. $(18,22,23,25,27,28,33,37,38,40,79-81,83-85,87-91,94,97,99)$ 
Table 2

Summary of included studies

\begin{tabular}{|c|c|c|c|c|c|c|c|}
\hline \multirow{2}{*}{$\begin{array}{l}\text { Citation, } \\
\text { study design, } \\
\text { location }\end{array}$} & \multirow{2}{*}{$\begin{array}{l}\mathrm{N} \\
\mathrm{C} / \mathrm{T}\end{array}$} & \multirow[t]{2}{*}{ Patient/infection description } & \multirow{2}{*}{$\begin{array}{l}\text { Disease } \\
\text { severity }\end{array}$} & \multirow[t]{2}{*}{$\mathrm{C} / \mathrm{T}$ treatment } & \multicolumn{3}{|c|}{ Outcome, \% (n/N) } \\
\hline & & & & & Clinical & Micro. & Mortality \\
\hline \multicolumn{8}{|l|}{2020 studies } \\
\hline \multicolumn{8}{|l|}{$\begin{array}{l}\text { Peer-reviewed } \\
\text { literature }\end{array}$} \\
\hline $\begin{array}{l}\text { Bassetti et al. } \\
2020(18) \\
\text { Retrospective, } \\
\text { multicenter } \\
\text { Italy }\end{array}$ & 153 & $\begin{array}{l}\text { ESBL-producing Enterobacterales } \\
\text { infections, including NP (30.0\%), } \\
\text { cUTI (22.2\%), and clAl (16.3\%). }\end{array}$ & $\begin{array}{l}>\mathrm{ICU} N= \\
74 \\
>\mathrm{CCl} \\
\text { mean }= \\
4.9\end{array}$ & $\begin{array}{l}\text { > Dose C/T: } \\
1.5 \mathrm{~g} \text { q8h (75.0; } \\
\text { of which } 6 \\
\text { patients received } \\
\text { creatinine } \\
\text { clearance } \\
\text { adjusted dose) } \\
\text { or } 3 \mathrm{~g} \text { q8h } \\
(24.8 \%) \\
\text { > Empiric C/T: } \\
30.0 \% \\
>\text { Confirmed C/T: } \\
70.0 \% \\
\text { > Duration: med. } \\
\text { (range): } 14 \text { (8- } \\
\text { 25) days }\end{array}$ & $\begin{array}{l}83.7 \\
(128 / 153)\end{array}$ & - & $\begin{array}{l}9.8 \\
(15 / 153)\end{array}$ \\
\hline $\begin{array}{l}\text { Bosaeed et } \\
\text { al. 2020(19) } \\
\\
\text { Retrospective, } \\
\text { single center } \\
\text { Saudi Arabia }\end{array}$ & 19 & $\begin{array}{l}\text { MDR PsA infections, including } \\
\text { NP (32\%), CLABSI (21\%), and } \\
\text { ABSSSI (16\%), and CIAI (16\%). }\end{array}$ & $\begin{array}{l}>1 C U N= \\
12\end{array}$ & $\begin{array}{l}\text { > Dose C/T: } \\
1.5 \mathrm{~g} \text { q8h } \\
(42.1 \%) \text { or } 3 \mathrm{~g} \\
\text { q8h }(10.5 \%) \text { or } \\
\text { creatinine } \\
\text { clearance } \\
\text { adjusted (47.4\%) } \\
\text { > Duration: med. } \\
\text { (range): } 14 \text { (7- } \\
\text { 35) days }\end{array}$ & $\begin{array}{l}95 \\
(18 / 19)\end{array}$ & $\begin{array}{l}74 \\
(14 / 19)\end{array}$ & $\begin{array}{l}21 \\
(4 / 19)\end{array}$ \\
\hline $\begin{array}{l}\text { Buonomo et } \\
\text { al. } 2020(20) \\
\text { Retrospective, } \\
\text { single center } \\
\text { case series } \\
\text { Italy }\end{array}$ & 4 & $\begin{array}{l}\text { PsA ( } 50 \% \text { MDR; } 50 \% \text { XDR) cSSTI in } \\
\text { patients with chronic kidney } \\
\text { disease. }\end{array}$ & - & $\begin{array}{l}\text { > Dose } \mathrm{C} / \mathrm{T}: \\
\text { creatine } \\
\text { clearance } \\
\text { adjusted } \\
(100.0 \%)- \\
0.75 \mathrm{~g} \text { q8h } \\
(75 \%), 0.375 \mathrm{~g} \\
\text { q8h }(25 \%) \\
\text { > Empiric } \mathrm{C} / \mathrm{T}: \\
0.0 \% \\
\text { > Confirmed } \mathrm{C} / \mathrm{T}: \\
100.0 \% \\
\text { > Duration: med. } \\
\text { (range): } 14 \text { (14) } \\
\text { days }\end{array}$ & $\begin{array}{l}100.0 \\
(4 / 4)\end{array}$ & - & 0 \\
\hline $\begin{array}{l}\text { Jones et al. } \\
2020(21) \\
\text { Retrospective } \\
\text { single center, } \\
\text { case series } \\
\text { US }\end{array}$ & 7 & $\begin{array}{l}\text { PsA ( } 57.1 \% \text { non-MDR; } 42.9 \% \text { MDR) } \\
\text { infections (one patient also had an } \\
\text { E. coli infection), including } \\
\text { pneumonia }(42.9 \%) \text {, cUTI }(28.6 \%) \text {, } \\
\text { and bacteremia }(14.3 \%) \text {. }\end{array}$ & - & $\begin{array}{l}\text { > Dose } \mathrm{C} / \mathrm{T} \text { : } \\
4.5 \mathrm{~g} \mathrm{qd}(\mathrm{Cl} ; \\
85.7 \%), 9 \mathrm{~g} \text { qd } \\
(\mathrm{Cl} ; 14.3 \%) \\
\text { > Duration: med. } \\
\text { (range): } 14 \text { (6- } \\
\text { 42) days }\end{array}$ & $\begin{array}{l}85.7 \\
(6 / 7)\end{array}$ & $\begin{array}{l}100.0 \\
(3 / 3)\end{array}$ & $0^{a}$ \\
\hline
\end{tabular}




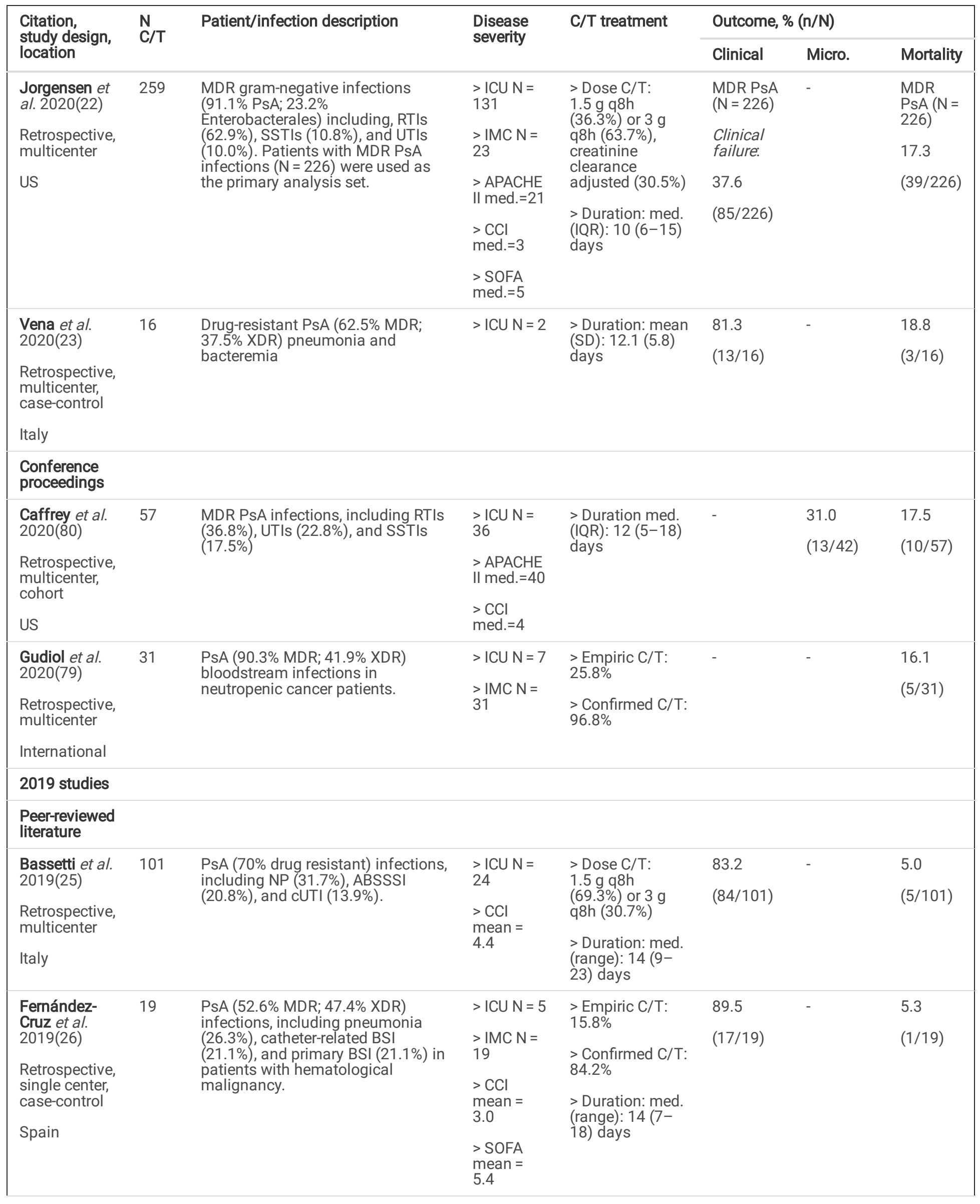




\begin{tabular}{|c|c|c|c|c|c|c|c|}
\hline \multirow{2}{*}{$\begin{array}{l}\text { Citation, } \\
\text { study design, } \\
\text { location }\end{array}$} & \multirow{2}{*}{$\begin{array}{l}\mathrm{N} \\
\mathrm{C} / \mathrm{T}\end{array}$} & \multirow[t]{2}{*}{ Patient/infection description } & \multirow{2}{*}{$\begin{array}{l}\text { Disease } \\
\text { severity }\end{array}$} & \multirow[t]{2}{*}{$\mathrm{C} / \mathrm{T}$ treatment } & \multicolumn{3}{|c|}{ Outcome, \% (n/N) } \\
\hline & & & & & Clinical & Micro. & Mortality \\
\hline \multirow{7}{*}{$\begin{array}{l}\text { Gerlach et al. } \\
\text { 2019(24) } \\
\text { Retrospective, } \\
\text { single center } \\
\text { US }\end{array}$} & \multirow[t]{7}{*}{18} & \multirow[t]{7}{*}{ MDR PsA osteomyelitis } & \multirow{2}{*}{$\begin{array}{l}>I C U N= \\
11\end{array}$} & \multirow{4}{*}{$\begin{array}{l}\text { > Dose C/T: } \\
1.5 \mathrm{~g} \mathrm{q8h} \\
(27.7 \%) \text { or } 3 \mathrm{~g} \\
\text { q8h }(55.6 \%) \text { or } \\
\text { creatinine } \\
\text { clearance } \\
\text { adjusted }(16.7 \%)\end{array}$} & \multirow{7}{*}{$\begin{array}{l}50.0 \\
(9 / 18)\end{array}$} & \multirow{7}{*}{$\begin{array}{l}75.0 \\
(3 / 4)\end{array}$} & \multirow{7}{*}{$\begin{array}{l}22.2 \\
(4 / 18)\end{array}$} \\
\hline & & & & & & & \\
\hline & & & \multirow{5}{*}{$\begin{array}{l}>\text { APACHE } \\
\text { II } \\
\text { med. }=13.5 \\
>\text { CCl } \\
\text { med. }=5.5\end{array}$} & & & & \\
\hline & & & & & & & \\
\hline & & & & $\begin{array}{l}>\text { Empiric } \mathrm{C} / \mathrm{T}: \\
0.0 \%\end{array}$ & & & \\
\hline & & & & $\begin{array}{l}>\text { Confirmed } \mathrm{C} / \mathrm{T}: \\
100.0 \%\end{array}$ & & & \\
\hline & & & & $\begin{array}{l}\text { > Duration: med. } \\
\text { (range): } 39 \text { (3- } \\
\text { 98) days }\end{array}$ & & & \\
\hline \multirow{5}{*}{$\begin{array}{l}\text { Pogue et al. } \\
2019(27) \\
\text { Retrospective, } \\
\text { multicenter, } \\
\text { case-control } \\
\text { US }\end{array}$} & \multirow[t]{5}{*}{100} & \multirow{5}{*}{$\begin{array}{l}\text { MDR or XDR PsA infections, } \\
\text { including NP (VABP [52.0\%], HABP } \\
[12.0 \%]) \text {, cUTIs (16.0\%), and wound } \\
(13.0 \%) \text {. }\end{array}$} & \multirow{2}{*}{$\begin{array}{l}>I C U N= \\
70\end{array}$} & \multirow{2}{*}{$\begin{array}{l}\text { > Dose C/T: } 3 \mathrm{~g} \\
\text { q8h (63\%), } 1.5 \mathrm{~g} \\
\text { q8h }(38 \%)\end{array}$} & \multirow{5}{*}{$\begin{array}{l}81.0 \\
(81 / 100)\end{array}$} & \multirow[t]{5}{*}{-} & \multirow{5}{*}{$\begin{array}{l}20.0 \\
(20 / 100)\end{array}$} \\
\hline & & & & & & & \\
\hline & & & & \multirow{3}{*}{$\begin{array}{l}\text { > Duration: med. } \\
\text { (IQR): } 9.5(7-14) \\
\text { days }\end{array}$} & & & \\
\hline & & & $\begin{array}{l}>\mathrm{CCl} \\
\text { mean }=3\end{array}$ & & & & \\
\hline & & & $>$ SOFA $=8$ & & & & \\
\hline \multirow{5}{*}{$\begin{array}{l}\text { Rodriguez- } \\
\text { Nunez et al. } \\
\text { 2019(28) } \\
\text { Retrospective, } \\
\text { multicenter } \\
\text { International }\end{array}$} & \multirow[t]{5}{*}{90} & \multirow{5}{*}{$\begin{array}{l}\text { Drug-resistant PsA RTIs }(76.7 \% \\
\text { XDR; } 23.3 \% \text { MDR). }\end{array}$} & \multirow{5}{*}{$\begin{array}{l}>\mathrm{CCl} \\
\text { med. }=5\end{array}$} & \multirow{4}{*}{$\begin{array}{l}\text { > Dose C/T: } \\
\text { standard }(1.5 \mathrm{~g} \\
\text { q8h or creatinine } \\
\text { clearance } \\
\text { adjusted; } 40 \%) \text {, } \\
\text { high }(3 \mathrm{~g} \text { q } 8 \mathrm{~h} \text { or } \\
\text { double creatinine } \\
\text { clearance } 60 \%) \text {. }\end{array}$} & \multirow{5}{*}{$\begin{array}{l}56.7 \\
(51 / 90)\end{array}$} & \multirow[t]{5}{*}{-} & 27.8 \\
\hline & & & & & & & $(25 / 90)$ \\
\hline & & & & & & & \\
\hline & & & & & & & \\
\hline & & & & $\begin{array}{l}\text { > Duration: med. } \\
\text { (IQR): } 14 \text { (10- } \\
\text { 16) days }\end{array}$ & & & \\
\hline Tan et al. & 5 & MDR gram-negative $(60 \%$ PsA; $40 \%$ & - & > Dose C/T: & 60.0 & - & 20.0 \\
\hline & & & & $3 \mathrm{~g} \mathrm{q} 8 \mathrm{~h}(80 \%)$ & $(3 / 5)$ & & $(1 / 5)$ \\
\hline $\begin{array}{l}\text { single center, } \\
\text { case series }\end{array}$ & & & & $\begin{array}{l}>\text { Empiric } \mathrm{C} / \mathrm{T} \text { : } \\
0 \%\end{array}$ & & & \\
\hline US & & & & $\begin{array}{l}>\text { Confirmed } \mathrm{C} / \mathrm{T}: \\
100 \%\end{array}$ & & & \\
\hline & & & & $\begin{array}{l}\text { > Duration mean: } \\
37.8 \text { days }\end{array}$ & & & \\
\hline $\begin{array}{l}\text { Conference } \\
\text { proceedings }\end{array}$ & & & & & & & \\
\hline $\begin{array}{l}\text { Cabrera et al. } \\
2019(85)\end{array}$ & 45 & $\begin{array}{l}\text { Gram-negative (84.4\% PsA; } 71.1 \% \\
\text { MDR PsA) infections, including }\end{array}$ & $\begin{array}{l}>1 C U N= \\
19\end{array}$ & $\begin{array}{l}\text { > Empiric } \mathrm{C} / \mathrm{T} \text { : } \\
21.7 \%\end{array}$ & 68.9 & - & 0 \\
\hline $\begin{array}{l}\text { Retrospective, } \\
\text { multicenter }\end{array}$ & & wound $(9 \%)$, and bone (9\%). & $\begin{array}{l}>\mathrm{IMCN}= \\
6\end{array}$ & $\begin{array}{l}\text { > Confirmed C/T: } \\
78.3 \%\end{array}$ & & & \\
\hline US & & & & $\begin{array}{l}\text { > Duration med. } \\
\text { (IQR): } 8(4-12) \\
\text { days }\end{array}$ & & & \\
\hline
\end{tabular}




\begin{tabular}{|c|c|c|c|c|c|c|c|}
\hline \multirow{2}{*}{$\begin{array}{l}\text { Citation, } \\
\text { study design, } \\
\text { location }\end{array}$} & \multirow{2}{*}{$\begin{array}{l}\mathrm{N} \\
\mathrm{C} / \mathrm{T}\end{array}$} & \multirow[t]{2}{*}{ Patient/infection description } & \multirow{2}{*}{$\begin{array}{l}\text { Disease } \\
\text { severity }\end{array}$} & \multirow[t]{2}{*}{$\mathrm{C} / \mathrm{T}$ treatment } & \multicolumn{3}{|c|}{ Outcome, \% (n/N) } \\
\hline & & & & & Clinical & Micro. & Mortality \\
\hline $\begin{array}{l}\text { Hart et al. } \\
\text { 2019(84) }\end{array}$ & \multirow[t]{4}{*}{70} & \multirow{4}{*}{$\begin{array}{l}\text { MDR PsA infections, including } \\
\text { pneumonia ( } 56 \%) \text {, wound ( } 11 \%), \text { IAI } \\
(10 \%) \text { in immunocompromised } \\
\text { patients. }\end{array}$} & $\begin{array}{l}>I C U N= \\
33\end{array}$ & \multirow{4}{*}{$\begin{array}{l}\text { > Duration mean } \\
\text { (SD): } 13(10.8) \\
\text { days }\end{array}$} & \multirow{4}{*}{$\begin{array}{l}69 \\
(48 / 70)\end{array}$} & \multirow[t]{4}{*}{-} & \multirow{4}{*}{$\begin{array}{l}19 \\
(13 / 70)\end{array}$} \\
\hline $\begin{array}{l}\text { Retrospective, } \\
\text { multicenter }\end{array}$ & & & $\begin{array}{l}>\mathrm{IMCN}= \\
70\end{array}$ & & & & \\
\hline \multirow[t]{2}{*}{ US } & & & $\begin{array}{l}>\text { APACHE } \\
\text { II med. }=18\end{array}$ & & & & \\
\hline & & & $\begin{array}{l}>\mathrm{CCl} \\
\text { med. }=5\end{array}$ & & & & \\
\hline $\begin{array}{l}\text { Mills et al. } \\
2019(83)\end{array}$ & \multirow[t]{3}{*}{62} & \multirow[t]{3}{*}{ MDR PsA pneumonia. } & $\begin{array}{l}>I C U N= \\
49\end{array}$ & \multirow[t]{3}{*}{$\begin{array}{l}\text { > Duration mean: } \\
16.1 \text { days }\end{array}$} & \multirow{3}{*}{$\begin{array}{l}72.6 \\
(45 / 62)\end{array}$} & \multirow[t]{3}{*}{-} & \multirow{3}{*}{$\begin{array}{l}29 \\
(18 / 62)\end{array}$} \\
\hline $\begin{array}{l}\text { Retrospective, } \\
\text { multicenter } \\
\text { cohort }\end{array}$ & & & $\begin{array}{l}>\mathrm{IMCN}= \\
13\end{array}$ & & & & \\
\hline US & & & & & & & \\
\hline $\begin{array}{l}\text { Sheffield et } \\
\text { al. 2019(82) }\end{array}$ & \multirow[t]{3}{*}{4} & \multirow{3}{*}{$\begin{array}{l}\text { PsA or ESBL-producing E. coli } \\
\text { infections, including LVAD infection } \\
(50.0 \%), \text { RTI }(25.0 \%) \text {, and IAI } \\
(25.0 \%) \text {. }\end{array}$} & - & $\begin{array}{l}\text { > Dose } \mathrm{C} / \mathrm{T} \\
\text { med.: } 6 \mathrm{~g} \mathrm{Cl} \text { qd }\end{array}$ & \multirow[t]{3}{*}{-} & \multirow[t]{3}{*}{-} & \multirow[t]{3}{*}{0} \\
\hline $\begin{array}{l}\text { Retrospective, } \\
\text { case series }\end{array}$ & & & & $\begin{array}{l}\text { > Duration range: } \\
6-91 \text { days }\end{array}$ & & & \\
\hline US & & & & & & & \\
\hline $\begin{array}{l}\text { Trisler et al. } \\
\text { 2019(81) }\end{array}$ & \multirow[t]{3}{*}{35} & \multirow{3}{*}{$\begin{array}{l}\text { PsA infections, including RTI } \\
(71.4 \%), \text { IAI ( } 14.3 \%) \text {, and } \\
\text { osteomyelitis (5.7\%) in patients } \\
\text { with and without CF. }\end{array}$} & \multirow[t]{3}{*}{-} & $\begin{array}{l}\text { > Empiric } \mathrm{C} / \mathrm{T} \text { : } \\
0.0 \%\end{array}$ & \multirow{3}{*}{$\begin{array}{l}\text { Clinical } \\
\text { failure: } \\
54.3 \\
(19 / 35)\end{array}$} & \multirow[t]{3}{*}{-} & \multirow[t]{3}{*}{-} \\
\hline $\begin{array}{l}\text { Retrospective, } \\
\text { multicenter }\end{array}$ & & & & $\begin{array}{l}\text { > Confirmed C/T: } \\
100.0 \%\end{array}$ & & & \\
\hline US & & & & $\begin{array}{l}\text { > Duration med. } \\
(\mathrm{IQR}): \mathrm{CF}=18.5 \\
(14-37.5) \text { days, } \\
\text { non-CF }=15.0 \\
(10-25) \text { days }\end{array}$ & & & \\
\hline \multicolumn{8}{|l|}{2018 studies } \\
\hline \multicolumn{8}{|l|}{$\begin{array}{l}\text { Peer-reviewed } \\
\text { literature }\end{array}$} \\
\hline $\begin{array}{l}\text { Diaz- } \\
\text { Cañestro et }\end{array}$ & 58 & PsA (86.2\% XDR) infections, & $>\operatorname{ICUN}=$ & > Dose C/T: & 63.8 & - & 27.6 \\
\hline al. 2018(30) & & $(17.2 \%)$, and IAls $(6.9 \%)$. & & $(46.6 \%), 3 \mathrm{~g} \mathrm{q} 8 \mathrm{~h}$ & $(37 / 58)$ & & $(16 / 58)$ \\
\hline Prospective, & & & $\begin{array}{l}>\operatorname{IMCN}= \\
7\end{array}$ & $\begin{array}{l}(41.4 \%), 0.15 \mathrm{~g} \\
\text { q8h }(12.1 \%)\end{array}$ & & & \\
\hline single center & & & $\begin{array}{l}>\mathrm{CCl} \\
\text { med. }=4\end{array}$ & $\begin{array}{l}\text { > Empiric } \mathrm{C} / \mathrm{T}: \\
1.7 \%\end{array}$ & & & \\
\hline Spain & & & $\begin{array}{l}>\text { SOFA } \\
\text { med. }=3\end{array}$ & $\begin{array}{l}\text { > Confirmed C/T: } \\
91.4 \%\end{array}$ & & & \\
\hline & & & & $\begin{array}{l}\text { > Duration mean } \\
\text { (SD): } 11.4(6.2) \\
\text { days }\end{array}$ & & & \\
\hline
\end{tabular}




\begin{tabular}{|c|c|c|c|c|c|c|c|}
\hline \multirow{2}{*}{$\begin{array}{l}\text { Citation, } \\
\text { study design, } \\
\text { location }\end{array}$} & \multirow{2}{*}{$\begin{array}{l}\mathrm{N} \\
\mathrm{C} / \mathrm{T}\end{array}$} & \multirow[t]{2}{*}{ Patient/infection description } & \multirow{2}{*}{$\begin{array}{l}\text { Disease } \\
\text { severity }\end{array}$} & \multirow[t]{2}{*}{$\mathrm{C} / \mathrm{T}$ treatment } & \multicolumn{3}{|c|}{ Outcome, \% (n/N) } \\
\hline & & & & & Clinical & Micro. & Mortality \\
\hline \multirow{2}{*}{$\begin{array}{l}\text { Dietl et al. } \\
\text { 2018(31) } \\
\text { Retrospective, } \\
\text { single center, } \\
\text { case series }\end{array}$} & \multirow[t]{5}{*}{7} & \multirow{5}{*}{$\begin{array}{l}\text { XDR PsA SSTIs (43\%) and } \\
\text { osteomyelitis (57\%). }\end{array}$} & \multirow{5}{*}{$\begin{array}{l}>\mathrm{CCl} \\
\text { med. }=6\end{array}$} & > Dose C/T: & 86 & 100 & 0 \\
\hline & & & & $\begin{array}{l}0.75 \mathrm{~g} \mathrm{q} 8 \mathrm{~h} \\
(29 \%), 0.375 \mathrm{~g} \\
\mathrm{q} 8 \mathrm{~h}(29 \%)\end{array}$ & $(6 / 7)$ & $(4 / 4)$ & \\
\hline \multirow[t]{3}{*}{ Spain } & & & & $\begin{array}{l}\text { > Empiric } \mathrm{C} / \mathrm{T}: \\
0 \%\end{array}$ & & & \\
\hline & & & & $\begin{array}{l}>\text { Confirmed } \mathrm{C} / \mathrm{T} \text { : } \\
71 \%\end{array}$ & & & \\
\hline & & & & $\begin{array}{l}\text { > Duration med. } \\
\text { (range): SSTI } 13 \\
(4-27) \text { / osteo. } \\
48(21-66) \text { days }\end{array}$ & & & \\
\hline \multirow{4}{*}{$\begin{array}{l}\text { Escolà-Vergé } \\
\text { et al. } \\
2018(32) \\
\text { Retrospective, } \\
\text { single center } \\
\text { Spain }\end{array}$} & \multirow[t]{4}{*}{38} & \multirow{4}{*}{$\begin{array}{l}\text { XDR PsA infections, including RTIs } \\
(36.8 \%) \text {, SSTIs }(15.8 \%) \text {, and UTIs } \\
(15.8 \%) \text {. }\end{array}$} & \multirow{4}{*}{$\begin{array}{l}>\mathrm{ICUN}= \\
12 \\
>\mathrm{CCl} \\
\text { med. }=3.5\end{array}$} & \multirow{4}{*}{$\begin{array}{l}\text { > Dose } \mathrm{C} / \mathrm{T}: 3 \mathrm{~g} \\
\text { q8h }(60.5 \%), \\
1.5 \mathrm{~g} \mathrm{q} 8 \mathrm{~h} \\
(39.5 \%) \\
\text { > Duration med. } \\
\text { (range): } 15.5 \text { (3- } \\
\text { 62) days }\end{array}$} & \multirow{4}{*}{$\begin{array}{l}68.4 \\
(26 / 38)\end{array}$} & \multirow{4}{*}{$\begin{array}{l}\text { Micro. } \\
\text { recur:: } \\
31.6 \\
(12 / 38)\end{array}$} & \multirow{4}{*}{$\begin{array}{l}13.2 \\
(5 / 38)\end{array}$} \\
\hline & & & & & & & \\
\hline & & & & & & & \\
\hline & & & & & & & \\
\hline \multirow{3}{*}{$\begin{array}{l}\text { Gallagher et } \\
\text { al. 2018(33) } \\
\text { Retrospective, } \\
\text { multicenter } \\
\text { US }\end{array}$} & \multirow[t]{3}{*}{205} & \multirow{3}{*}{$\begin{array}{l}\text { MDR PsA infections, including 59\% } \\
\text { pneumonia, UTI (13.7\%), and } \\
\text { wound (12.7\%). }\end{array}$} & $\begin{array}{l}>I C U N= \\
105\end{array}$ & \multirow{3}{*}{$\begin{array}{l}\text { > Dose } \mathrm{C} / \mathrm{T}: 3 \mathrm{~g} \\
\text { q8h }(47.3 \%), \\
1.5 \mathrm{~g} \mathrm{q} 8 \mathrm{~h} \\
(52.7 \%) \\
\text { > Duration med. } \\
\text { (IQR): } 10(7-14) \\
\text { days }\end{array}$} & \multirow{3}{*}{$\begin{array}{l}73.7 \\
(151 / 205)\end{array}$} & \multirow{3}{*}{$\begin{array}{l}70.7 \\
(145 / 205)\end{array}$} & \multirow{3}{*}{$\begin{array}{l}19.0 \\
(39 / 205)\end{array}$} \\
\hline & & & $\begin{array}{l}>\text { APACHE } \\
\text { II med. }=19\end{array}$ & & & & \\
\hline & & & $\begin{array}{l}>\mathrm{CCl} \\
\text { med. }=4\end{array}$ & & & & \\
\hline \multirow{4}{*}{$\begin{array}{l}\text { Hakki et al. } \\
2018(34) \\
\text { Retrospective, } \\
\text { single center, } \\
\text { case series } \\
\text { US }\end{array}$} & \multirow[t]{4}{*}{6} & \multirow{4}{*}{$\begin{array}{l}7 \text { episodes of MDR PsA infections, } \\
\text { including bacteremia ( } 42.9 \%) \\
\text { pneumonia }(42.9 \%) \text {, and soft tissue } \\
(14.3 \%) \text { in patients with } \\
\text { hematological malignancy or } \\
\text { hematopoietic stem cell transplant. }\end{array}$} & \multirow[t]{4}{*}{$\begin{array}{l}>\mathrm{IMCN}= \\
6\end{array}$} & $\begin{array}{l}>\text { Dose } C / T: 3 \mathrm{~g} \\
\text { q8h }(100 \%)\end{array}$ & \multirow{4}{*}{$\begin{array}{l}71.4 \\
(5 / 7)^{\mathrm{b}}\end{array}$} & \multirow[t]{4}{*}{-} & \multirow[t]{4}{*}{0} \\
\hline & & & & $\begin{array}{l}\text { > Empiric } \mathrm{C} / \mathrm{T} \text { : } \\
33.3 \%\end{array}$ & & & \\
\hline & & & & $\begin{array}{l}\text { > Confirmed } \mathrm{C} / \mathrm{T}: \\
66.7 \%\end{array}$ & & & \\
\hline & & & & $\begin{array}{l}\text { > Duration med. } \\
\text { (range): } 29(14- \\
\text { 103) days }\end{array}$ & & & \\
\hline $\begin{array}{l}\text { Xipell et al. } \\
2018(35)\end{array}$ & 23 & $\begin{array}{l}24 \text { episodes of MDR PsA infections, } \\
\text { including RTI }(33.3 \%) \text {, UTI }(29.2 \%) \text {. }\end{array}$ & $>\mathrm{ICUN}=4$ & $\begin{array}{l}\text { > Dose } \mathrm{C} / \mathrm{T}: 3 \mathrm{~g} \\
\text { q } 8 \mathrm{~h} \text { or } 1.25 \mathrm{~g}\end{array}$ & 88 & 75 & 22 \\
\hline Retrospective, & & and SSTI $(25.0 \%)$. & & $\begin{array}{l}\text { q8h or } 0.75 \mathrm{~g} \\
\mathrm{q} 8 \mathrm{~h}(\%=\mathrm{NR})\end{array}$ & $(21 / 24)$ & $(12 / 16)$ & $(5 / 23)$ \\
\hline case series & & & & $\begin{array}{l}\text { > Empiric C/T: } \\
13 \%\end{array}$ & & & \\
\hline Spaimi & & & & $\begin{array}{l}>\text { Confirmed } \mathrm{C} / \mathrm{T} \text { : } \\
87 \%\end{array}$ & & & \\
\hline & & & & $\begin{array}{l}\text { > Duration mean } \\
\text { (SD): } 14.3(9.4) \\
\text { days }\end{array}$ & & & \\
\hline $\begin{array}{l}\text { Conference } \\
\text { proceedings }\end{array}$ & & & & & & & \\
\hline
\end{tabular}




\begin{tabular}{|c|c|c|c|c|c|c|c|}
\hline \multirow{2}{*}{$\begin{array}{l}\text { Citation, } \\
\text { study design, } \\
\text { location }\end{array}$} & \multirow{2}{*}{$\begin{array}{l}\mathrm{N} \\
\mathrm{C} / \mathrm{T}\end{array}$} & \multirow[t]{2}{*}{ Patient/infection description } & \multirow{2}{*}{$\begin{array}{l}\text { Disease } \\
\text { severity }\end{array}$} & \multirow[t]{2}{*}{$\mathrm{C} / \mathrm{T}$ treatment } & \multicolumn{3}{|c|}{ Outcome, \% (n/N) } \\
\hline & & & & & Clinical & Micro. & Mortality \\
\hline \multirow{2}{*}{$\begin{array}{l}\text { Elabor et al. } \\
\text { 2018(97) }\end{array}$} & \multirow[t]{3}{*}{65} & \multirow{3}{*}{$\begin{array}{l}\text { MDR PsA infections, including } \\
\text { pneumonia, wound/bone/joint } \\
\text { infections, UTIs, and IAls (\% NR) in } \\
\text { immunocompromised patients. }\end{array}$} & \multirow{2}{*}{$\begin{array}{l}>1 \mathrm{CU} N= \\
37\end{array}$} & \multirow{5}{*}{$\begin{array}{l}\text { > Dose } \mathrm{C} / \mathrm{T}: 3 \mathrm{~g} \\
\text { q8h }(35.4 \%), \\
1.5 \mathrm{~g} \mathrm{q} 8 \mathrm{~h} \\
(35.4 \%),<1.5 \mathrm{~g} \\
\text { q8h }(29.2 \%)\end{array}$} & 78.4 & 75.3 & 13.9 \\
\hline & & & & & $(51 / 65)$ & (NR) & $(9 / 65)$ \\
\hline $\begin{array}{l}\text { Retrospective, } \\
\text { multicenter }\end{array}$ & & & $\begin{array}{l}>\operatorname{IMCN}= \\
65\end{array}$ & & & & \\
\hline \multirow[t]{2}{*}{ US } & & & $\begin{array}{l}>\text { APACHE } \\
\text { II med. }=20\end{array}$ & & & & \\
\hline & & & $\begin{array}{l}>\mathrm{CCl} \\
\text { med. }=6\end{array}$ & & & & \\
\hline \multirow{2}{*}{$\begin{array}{l}\text { Gioia et al. } \\
\text { 2018(96) }\end{array}$} & \multirow[t]{4}{*}{15} & \multirow{4}{*}{$\begin{array}{l}\text { MDR PsA infections, including RTI } \\
(53 \%) \text {, IAI }(27 \%) \text {, and wound }(13 \%) \text {. }\end{array}$} & $>\mathrm{ICUN}=8$ & \multirow{3}{*}{$\begin{array}{l}\text { > Dose C/T: } \\
1.5 \mathrm{~g} \mathrm{q} 8 \mathrm{~h}(67 \%) \\
<1.5 \mathrm{~g} \mathrm{q} 8 \mathrm{~h} \\
(13 \%), 3 \mathrm{~g} \mathrm{q} 8 \mathrm{~h} \\
(20 \%)\end{array}$} & 60 & 60 & 27 \\
\hline & & & \multirow{2}{*}{$>{ }_{9} \mathrm{IMCN}=$} & & \multirow[t]{3}{*}{$(9 / 15)$} & \multirow[t]{3}{*}{$(9 / 15)$} & $(4 / 15)$ \\
\hline $\begin{array}{l}\text { Retrospective, } \\
\text { single center }\end{array}$ & & & & & & & \\
\hline Spain & & & $\begin{array}{l}>\mathrm{CCl} \\
\text { med. }=4\end{array}$ & $\begin{array}{l}\text { > Duration med. } \\
\text { (range): } 23 \text { (2- } \\
\text { 102) days }\end{array}$ & & & \\
\hline \multirow{2}{*}{$\begin{array}{l}\text { Henry et al. } \\
\text { 2018(95) }\end{array}$} & \multirow[t]{6}{*}{29} & \multirow{6}{*}{$\begin{array}{l}42 \text { treatment courses for gram- } \\
\text { negative infections ( } 86 \% \text { PsA; } 7 \% \\
\text { Klebsiella spp.; } 7 \% \text { E. colli), } \\
\text { including pneumonia }(26 \%) \text {, IAls } \\
\text { (21\%), and UTI ( } 21 \%) \text {. }\end{array}$} & \multirow{6}{*}{$\begin{array}{l}>\mathrm{ICU} N= \\
15\end{array}$} & > Dose $\mathrm{C} / \mathrm{T}$ : & \multirow{6}{*}{$\begin{array}{l}76 \\
(32 / 42)\end{array}$} & \multirow[t]{6}{*}{-} & \multirow{6}{*}{$\begin{array}{l}38 \\
(11 / 29)\end{array}$} \\
\hline & & & & $1.5 \mathrm{~g}(0.15-3 \mathrm{~g})$ & & & \\
\hline $\begin{array}{l}\text { Retrospective, } \\
\text { sinqle center }\end{array}$ & & & & q8h & & & \\
\hline \multirow[t]{3}{*}{ US } & & & & $\begin{array}{l}>\text { Empiric } \mathrm{C} / \mathrm{T} \text { : } \\
36 \%\end{array}$ & & & \\
\hline & & & & $\begin{array}{l}>\text { Confirmed } \mathrm{C} / \mathrm{T} \text { : } \\
64 \%\end{array}$ & & & \\
\hline & & & & $\begin{array}{l}\text { > Duration med. } \\
\text { (range): } 10 \text { ( } 2- \\
\text { 85) days }\end{array}$ & & & \\
\hline \multirow{6}{*}{$\begin{array}{l}\text { Hirsch et al. } \\
\text { 2018(94) } \\
\text { Retrospective, } \\
\text { multicenter } \\
\text { US }\end{array}$} & \multirow[t]{6}{*}{35} & $\begin{array}{l}\text { Gram-negative infections (79\% PsA: } \\
60.7 \% \text { MDR; } 21.4 \% \text { XDR), including }\end{array}$ & $\begin{array}{l}>I C U N= \\
26\end{array}$ & $\begin{array}{l}>\text { Dose } \mathrm{C} / \mathrm{T}: 3 \mathrm{~g} \\
\text { q } 8 \mathrm{~h}(42.9 \%)\end{array}$ & 77.4 & 74.2 & 14.3 \\
\hline & & RTIs $(33 \%)$, BSIs $(21 \%)$, and & & $1.5 \mathrm{~g} \mathrm{q} 8 \mathrm{~h}$ & $(24 / 31)$ & $(23 / 31)$ & $(5 / 35)$ \\
\hline & & & & $\begin{array}{l}(31.4 \%), 0.75 \mathrm{~g} \\
\mathrm{q} 8 \mathrm{~h}(17.1 \%),\end{array}$ & & & \\
\hline & & & & $\begin{array}{l}(2.9 \%), \text { Other } \\
(5.7 \%)\end{array}$ & & & \\
\hline & & & & $\begin{array}{l}\text { > Empiric } \mathrm{C} / \mathrm{T} \text { : } \\
20 \%\end{array}$ & & & \\
\hline & & & & $\begin{array}{l}>\text { Confirmed } \mathrm{C} / \mathrm{T} \text { : } \\
80 \%\end{array}$ & & & \\
\hline Jayakumar et & 22 & PsA (95\%; $90 \%$ MDR) sepsis and/or & - & > Dose C/T: $3 \mathrm{~g}$ & 77 & - & 23 \\
\hline & & & & $(45 \%)$ & $(17 / 22)$ & & $(5 / 22)$ \\
\hline single center & & & & $\begin{array}{l}\text { > Empiric } \mathrm{C} / \mathrm{T} \text { : } \\
18 \%\end{array}$ & & & \\
\hline US & & & & $\begin{array}{l}>\text { Confirmed } \mathrm{C} / \mathrm{T} \text { : } \\
82 \%\end{array}$ & & & \\
\hline & & & & $\begin{array}{l}\text { > Duration med: } \\
10 \text { days }\end{array}$ & & & \\
\hline
\end{tabular}




\begin{tabular}{|c|c|c|c|c|c|c|c|}
\hline \multirow{2}{*}{$\begin{array}{l}\text { Citation, } \\
\text { study design, } \\
\text { location }\end{array}$} & \multirow{2}{*}{$\begin{array}{l}\mathrm{N} \\
\mathrm{C} / \mathrm{T}\end{array}$} & \multirow[t]{2}{*}{ Patient/infection description } & \multirow{2}{*}{$\begin{array}{l}\text { Disease } \\
\text { severity }\end{array}$} & \multirow[t]{2}{*}{$\mathrm{C} / \mathrm{T}$ treatment } & \multicolumn{3}{|c|}{ Outcome, \% (n/N) } \\
\hline & & & & & Clinical & Micro. & Mortality \\
\hline $\begin{array}{l}\text { Jorgensen et } \\
\text { al. 2018(90) }\end{array}$ & \multirow[t]{4}{*}{116} & \multirow{4}{*}{$\begin{array}{l}\text { MDR PsA infections, including RTI } \\
(65 \%), \text { UTI (10.3\%), and SSTI } \\
(9.4 \%) \text {. }\end{array}$} & $\begin{array}{l}>\mathrm{ICUN}= \\
72\end{array}$ & \multirow[t]{4}{*}{-} & \multirow{4}{*}{$\begin{array}{l}\text { Clinical } \\
\text { failure: } \\
\\
38.8 \\
(45 / 116)\end{array}$} & \multirow[t]{4}{*}{-} & \multirow{4}{*}{$\begin{array}{l}17.2 \\
(20 / 116)\end{array}$} \\
\hline $\begin{array}{l}\text { Retrospective, } \\
\text { multicenter }\end{array}$ & & & $\begin{array}{l}>\mathrm{IMCN}= \\
22\end{array}$ & & & & \\
\hline \multirow[t]{2}{*}{ US } & & & $\begin{array}{l}>\text { APACHE } \\
\text { II med. }=21\end{array}$ & & & & \\
\hline & & & $\begin{array}{l}>\mathrm{CCl} \\
\text { med. }=3.5\end{array}$ & & & & \\
\hline $\begin{array}{l}\text { Jorgensen et } \\
\text { al. 2018(91) }\end{array}$ & \multirow[t]{3}{*}{137} & \multirow[t]{3}{*}{ MDR PsA infections } & $\begin{array}{l}>1 \mathrm{ICU} N= \\
87\end{array}$ & \multirow[t]{3}{*}{-} & \multirow[t]{3}{*}{-} & \multirow[t]{3}{*}{-} & \multirow{3}{*}{$\begin{array}{l}18.2 \\
(25 / 137)\end{array}$} \\
\hline $\begin{array}{l}\text { Retrospective, } \\
\text { multicenter }\end{array}$ & & & $\begin{array}{l}>1 \mathrm{IMCN}= \\
11\end{array}$ & & & & \\
\hline \multicolumn{2}{|l|}{ US } & & & & & & \\
\hline $\begin{array}{l}\text { Pogue et al. } \\
2018(89)\end{array}$ & \multirow[t]{2}{*}{113} & \multirow[t]{2}{*}{ PsA cUTI (64\%) and clAl (36\%). } & \multirow[t]{2}{*}{-} & $\begin{array}{l}\text { > Empiric } \mathrm{C} / \mathrm{T} \text { : } \\
31 \%\end{array}$ & \multirow[t]{2}{*}{-} & \multirow[t]{2}{*}{-} & \multirow{2}{*}{$\begin{array}{l}12.4 \\
(14 / 113)\end{array}$} \\
\hline $\begin{array}{l}\text { Retrospective, } \\
\text { multicenter } \\
\text { US }\end{array}$ & & & & $\begin{array}{l}\text { > Confirmed C/T: } \\
\text { early definite } \\
28 \% \text { and late } \\
\text { definite } 41 \%\end{array}$ & & & \\
\hline $\begin{array}{l}\text { Puzniak et al. } \\
\text { 2018(87) }\end{array}$ & \multirow[t]{3}{*}{1,490} & \multirow[t]{3}{*}{$\begin{array}{l}\text { Gram-negative infections ( } 78 \% \text { PsA } \\
{[202 / 259 \text { patients with }} \\
\text { microbiological results]). }\end{array}$} & \multirow{3}{*}{$\begin{array}{l}>\mathrm{ICUN}= \\
824 \\
>\mathrm{CCl} \\
\text { mean }=3\end{array}$} & \multirow[t]{3}{*}{-} & - & - & 9.1 \\
\hline $\begin{array}{l}\text { Retrospective, } \\
\text { multicenter }\end{array}$ & & & & & & & \\
\hline US & & & & & & & \\
\hline $\begin{array}{l}\text { Puzniak et al. } \\
2018(88)^{c}\end{array}$ & 199 & $\begin{array}{l}\text { PsA infections, including RTIs } \\
(57 \%) \text { and UTIs (17\%). }\end{array}$ & $\begin{array}{l}>\operatorname{ICUN}= \\
107\end{array}$ & $\begin{array}{l}\text { > Empiric } \mathrm{C} / \mathrm{T} \text { : } \\
34 \%\end{array}$ & - & - & \\
\hline $\begin{array}{l}\text { Retrospective, } \\
\text { multicenter } \\
\text { US }\end{array}$ & & & $\begin{array}{l}>\mathrm{CCl} \\
\text { mean }= \\
2.9\end{array}$ & $\begin{array}{l}\text { > Confirmed } \mathrm{C} / \mathrm{T} \text { : } \\
\text { early direct } 50 \% \\
\text { and late direct } \\
16 \%\end{array}$ & & & (עבוד/ \\
\hline & & & & $\begin{array}{l}\text { > Duration med. } \\
\text { (IQR): } 8(4-13) \\
\text { days }\end{array}$ & & & \\
\hline $\begin{array}{l}\text { Tordato et al. } \\
2018(86)\end{array}$ & 11 & $\begin{array}{l}\text { PsA infections (73\% XDR), } \\
\text { including RTIs (54\%). BSIs (27\%) }\end{array}$ & $>\operatorname{ICUN}=6$ & $\begin{array}{l}\text { > Duration med. } \\
\text { (range): } 16(6-\end{array}$ & 100.0 & - & $36.4^{d}$ \\
\hline Retrospective, & & and IAls $(18 \%)$. & $\begin{array}{l}>\mathrm{IMCN}= \\
3\end{array}$ & 27) days & $(11 / 11)$ & & $(4 / 11)$ \\
\hline Italy & & & $\begin{array}{l}>\mathrm{CCl} \\
\text { med. }=4\end{array}$ & & & & \\
\hline 2017 studies & & & & & & & \\
\hline $\begin{array}{l}\text { Peer-reviewed } \\
\text { literature }\end{array}$ & & & & & & & \\
\hline
\end{tabular}




\begin{tabular}{|c|c|c|c|c|c|c|c|}
\hline \multirow{2}{*}{$\begin{array}{l}\text { Citation, } \\
\text { study design, } \\
\text { location }\end{array}$} & \multirow{2}{*}{$\begin{array}{l}\mathrm{N} \\
\mathrm{C} / \mathrm{T}\end{array}$} & \multirow[t]{2}{*}{ Patient/infection description } & \multirow{2}{*}{$\begin{array}{l}\text { Disease } \\
\text { severity }\end{array}$} & \multirow[t]{2}{*}{$\mathrm{C} / \mathrm{T}$ treatment } & \multicolumn{3}{|c|}{ Outcome, \% (n/N) } \\
\hline & & & & & Clinical & Micro. & Mortality \\
\hline \multirow{2}{*}{$\begin{array}{l}\text { Alvarez } \\
\text { Lerma et al. } \\
2017(36)\end{array}$} & \multirow[t]{6}{*}{2} & \multirow{6}{*}{$\begin{array}{l}\text { PDR PsA ventilation-associated } \\
\text { respiratory infections }\end{array}$} & $>\operatorname{ICUN}=2$ & \multirow{3}{*}{$\begin{array}{l}\text { > Dose } \mathrm{C} / \mathrm{T}: \\
1.5 \mathrm{~g} \mathrm{q} 8 \mathrm{~h} \text { then } \\
0.75 \mathrm{~g} \mathrm{q} 8 \mathrm{~h} \\
(50 \%), 0.75 \mathrm{~g} \\
\text { q8h }(50 \%)\end{array}$} & 100 & 100 & 50 \\
\hline & & & \multirow{5}{*}{$\begin{array}{l}>\text { APACHE } \\
\text { II mean }= \\
25.5\end{array}$} & & $(2 / 2)$ & $(2 / 2)$ & $(1 / 2)$ \\
\hline \multirow{4}{*}{$\begin{array}{l}\text { Retrospective, } \\
\text { single center, } \\
\text { case series } \\
\text { Spain }\end{array}$} & & & & & & & \\
\hline & & & & $\begin{array}{l}\text { > Empiric } \mathrm{C} / \mathrm{T}: \\
0 \%\end{array}$ & & & \\
\hline & & & & $\begin{array}{l}\text { > Confirmed C/T: } \\
100 \%\end{array}$ & & & \\
\hline & & & & $\begin{array}{l}\text { > Duration: } \\
\text { mean }=15.5 \\
\text { days }\end{array}$ & & & \\
\hline \multirow{2}{*}{$\begin{array}{l}\text { Castón et al. } \\
\text { 2017(37) }\end{array}$} & \multirow[t]{6}{*}{12} & \multirow{6}{*}{$\begin{array}{l}\text { MDR PsA infections, including RTIs } \\
(50 \%) \text { and IAls }(25.0 \%) .83 \% \text { of } \\
\text { patients had septic shock. }\end{array}$} & \multirow{6}{*}{$\begin{array}{l}>\mathrm{IMCN}= \\
4\end{array}$} & > Dose $\mathrm{C} / \mathrm{T}$ : & 75.0 & 63.6 & 25.0 \\
\hline & & & & $\begin{array}{l}1.5 \mathrm{~g} \mathrm{gonh}(3 \% \%) \\
3 \mathrm{~g} \mathrm{q} 8 \mathrm{~h}(33 \%)\end{array}$ & $(9 / 12)$ & $(7 / 11)$ & $(3 / 12)$ \\
\hline $\begin{array}{l}\text { Retrospective, } \\
\text { multicenter, }\end{array}$ & & & & $\begin{array}{l}\text { > Empiric C/T: } \\
0 \%\end{array}$ & & & \\
\hline case series & & & & \multirow{2}{*}{$\begin{array}{l}\text { > Confirmed } \mathrm{C} / \mathrm{T} \text { : } \\
100 \%\end{array}$} & & & \\
\hline \multirow[t]{2}{*}{ Spain } & & & & & & & \\
\hline & & & & $\begin{array}{l}\text { > Duration med. } \\
\text { (range): } 12 \text { ( } 9- \\
\text { 18) days }\end{array}$ & & & \\
\hline \multirow{2}{*}{$\begin{array}{l}\text { Dinh et al. } \\
\text { 2017(38) }\end{array}$} & \multirow[t]{3}{*}{15} & \multirow{3}{*}{$\begin{array}{l}\text { XDR PsA infections, including RTIs } \\
(46.7 \%), \text { UTIs (20.0\%), and IAls } \\
(13.3 \%) \text {. }\end{array}$} & $>\operatorname{ICUN}=8$ & \multirow{2}{*}{$\begin{array}{l}\text { > Dose } \mathrm{C} / \mathrm{T}: \\
\text { med. }(\text { range })= \\
6 \mathrm{~g}(3-7.5 \mathrm{~g})\end{array}$} & 67 & 75 & 27 \\
\hline & & & $>\mathrm{IMCN}=$ & & $(10 / 15)$ & $(6 / 8)$ & $(4 / 15)$ \\
\hline $\begin{array}{l}\text { multicenter, } \\
\text { case series } \\
\text { France }\end{array}$ & & & $\begin{array}{l}>\text { SOFA } \\
\text { mean = } \\
7.6\end{array}$ & $\begin{array}{l}\text { > Duration med. } \\
\text { (range): } 15(4- \\
63) \text { days }\end{array}$ & & & \\
\hline $\begin{array}{l}\text { Haidar et al. } \\
2017(39)\end{array}$ & \multirow[t]{3}{*}{21} & \multirow{3}{*}{$\begin{array}{l}\text { MDR PsA infections, including } 86 \% \\
\text { RTIs, } 5 \% \text { cUTIs, } 5 \% \text { clAls, and } 5 \% \\
\text { bacteremia. }\end{array}$} & $\begin{array}{l}>\mathrm{IMCN}= \\
9\end{array}$ & \multirow{2}{*}{$\begin{array}{l}\text { > Dose C/T: } \\
1.5 \mathrm{~g} \mathrm{q} 8 \mathrm{~h}(48 \%), \\
0.75 \mathrm{~g} \mathrm{q} 8 \mathrm{~h} \\
(24 \%), 0.375 \mathrm{~g} \\
\text { q8h (5\%), Other } \\
(23 \%)\end{array}$} & $\begin{array}{l}\text { Clinical } \\
\text { failure: }\end{array}$ & - & 10 \\
\hline $\begin{array}{l}\text { Retrospective, } \\
\text { single center, } \\
\text { case series }\end{array}$ & & & $\begin{array}{l}>\mathrm{CCl} \\
\text { med. }=5\end{array}$ & & \multirow[t]{2}{*}{$\begin{array}{l}29 \\
(6 / 21)\end{array}$} & & $(2 / 21)$ \\
\hline US & & & $\begin{array}{l}>\text { SOFA } \\
\text { med. }=6\end{array}$ & $\begin{array}{l}\text { > Duration med. } \\
\text { (range): } 14 \text { (3- } \\
\text { 52) days }\end{array}$ & & & \\
\hline \multirow{2}{*}{$\begin{array}{l}\text { Munita et al. } \\
\text { 2017(40) }\end{array}$} & 35 & $\begin{array}{l}\text { CR PsA infections, including } \\
\text { pneumonia (51.0\%) and secondary }\end{array}$ & $\begin{array}{l}>\mathrm{CCl} \\
\text { med. }=4\end{array}$ & $\begin{array}{l}\text { > Dose C/T: } 3 \mathrm{~g} \\
\text { q } 8 \mathrm{~h}(26 \%)\end{array}$ & 74 & 100 & 22.8 \\
\hline & & BSI (17.1\%). & & $\begin{array}{l}0.375-1.25 \mathrm{~g} \\
\mathrm{q} 8 \mathrm{~h}(\%=\mathrm{NR})\end{array}$ & $(26 / 35)$ & $(25 / 25)$ & $(8 / 35)$ \\
\hline $\begin{array}{l}\text { mul } \\
\text { uS }\end{array}$ & & & & $\begin{array}{l}\text { > Duration med. } \\
\text { (range): } 16 \text { ( } 5- \\
\text { 27) days }\end{array}$ & & & \\
\hline
\end{tabular}




\begin{tabular}{|c|c|c|c|c|c|c|c|}
\hline \multirow{2}{*}{$\begin{array}{l}\text { Citation, } \\
\text { study design, } \\
\text { location }\end{array}$} & \multirow{2}{*}{$\begin{array}{l}\mathrm{N} \\
\mathrm{C} / \mathrm{T}\end{array}$} & \multirow[t]{2}{*}{ Patient/infection description } & \multirow{2}{*}{$\begin{array}{l}\text { Disease } \\
\text { severity }\end{array}$} & \multirow[t]{2}{*}{$\mathrm{C} / \mathrm{T}$ treatment } & \multicolumn{3}{|c|}{ Outcome, \% (n/N) } \\
\hline & & & & & Clinical & Micro. & Mortality \\
\hline \multirow{7}{*}{$\begin{array}{l}\text { Sacha et al. } \\
2017(41) \\
\text { Retrospective, } \\
\text { single center } \\
\text { US }\end{array}$} & \multirow[t]{7}{*}{49} & \multirow{7}{*}{$\begin{array}{l}60 \text { courses of therapy for gram- } \\
\text { negative infections ( } 86.7 \% \text { PsA: } \\
34.6 \% \text { non-MDR; } 40.4 \% \text { MDR; } 25.0 \% \\
\text { XDR), including NP (56.7\%), IAl } \\
\text { (18.3\%), and bacteremia (6.7\%) }\end{array}$} & \multirow{7}{*}{$\begin{array}{l}>1 \mathrm{ICUN}= \\
37 \\
>\mathrm{IMCN}= \\
25\end{array}$} & \multirow{4}{*}{$\begin{array}{l}\text { > Dose C/T: } 3 \mathrm{~g} \\
\text { q8h }(1.7 \%), 1.5 \mathrm{~g} \\
\text { q8h (51.7\%), } \\
0.75 \mathrm{~g} \text { q8h } \\
(26.7 \%), 0.375 \mathrm{~g} \\
\text { q8h (8.3\%), } \\
0.15 \mathrm{~g} \text { q8h } \\
(11.7 \%)\end{array}$} & \multirow{7}{*}{$\begin{array}{l}64.1^{f} \\
(25 / 39)\end{array}$} & \multirow{7}{*}{$\begin{array}{l}38.5 \\
(5 / 13)\end{array}$} & \multirow{7}{*}{$\begin{array}{l}16.7 \\
(10 / 60)\end{array}$} \\
\hline & & & & & & & \\
\hline & & & & & & & \\
\hline & & & & & & & \\
\hline & & & & $\begin{array}{l}\text { > Empiric } \mathrm{C} / \mathrm{T}: \\
36.7 \%\end{array}$ & & & \\
\hline & & & & $\begin{array}{l}\text { > Confirmed C/T: } \\
63.3 \%\end{array}$ & & & \\
\hline & & & & $\begin{array}{l}>\text { Duration med.: } \\
1-8 \text { days }^{\mathrm{e}}\end{array}$ & & & \\
\hline \multirow{4}{*}{$\begin{array}{l}\text { Xipell et al. } \\
2017(42) \\
\\
\text { Retrospective, } \\
\text { single center, } \\
\text { case series } \\
\text { Spain }\end{array}$} & \multirow[t]{4}{*}{3} & \multirow{4}{*}{$\begin{array}{l}\text { MDR or XDR PsA infections, } \\
\text { including mediastinitis, liver } \\
\text { abscess, and septic shock. }\end{array}$} & \multirow[t]{4}{*}{-} & $\begin{array}{l}\text { > Dose C/T: } \\
1.5 \mathrm{~g} \mathrm{q} 8 \mathrm{~h}(100 \%)\end{array}$ & \multirow{4}{*}{$\begin{array}{l}100 \\
(3 / 3)\end{array}$} & \multirow[t]{4}{*}{-} & \multirow[t]{4}{*}{0} \\
\hline & & & & $\begin{array}{l}>\text { Empiric } \mathrm{C} / \mathrm{T} \text { : } \\
0 \%\end{array}$ & & & \\
\hline & & & & $\begin{array}{l}>\text { Confirmed C/T: } \\
100 \%\end{array}$ & & & \\
\hline & & & & $\begin{array}{l}\text { > Duration mean } \\
\text { (range): } 30.3 \\
(21-42) \text { days }\end{array}$ & & & \\
\hline \multicolumn{8}{|l|}{$\begin{array}{l}\text { Conference } \\
\text { proceedings }\end{array}$} \\
\hline \multirow{5}{*}{$\begin{array}{l}\text { Leuthner et } \\
\text { al. 2017(98) } \\
\text { Retrospective, } \\
\text { single center } \\
\text { US }\end{array}$} & \multirow[t]{5}{*}{30} & \multirow{5}{*}{$\begin{array}{l}\text { Gram-negative infections (93\% PsA; } \\
3 \% \text { E. coli; } 3 \% \text { P stuartii), including } \\
\text { RTIs }(67 \%) \text {, cUTIs }(27 \%) \text {, and BSIs } \\
(20 \%) \text {. }\end{array}$} & \multirow{5}{*}{$\begin{array}{l}>\operatorname{ICUN}=8 \\
>\operatorname{IMCN}= \\
4\end{array}$} & \multirow{2}{*}{$\begin{array}{l}\text { > Dose C/T: } 3 \mathrm{~g} \\
\text { q8h ( } 57 \%), \text { Other } \\
(43 \%)\end{array}$} & \multirow{5}{*}{$\begin{array}{l}80 \\
(24 / 30)\end{array}$} & \multirow{5}{*}{$\begin{array}{l}92 \\
(11 / 12)\end{array}$} & \multirow{5}{*}{$\begin{array}{l}20 \\
(6 / 30)\end{array}$} \\
\hline & & & & & & & \\
\hline & & & & $\begin{array}{l}>\text { Empiric } \mathrm{C} / \mathrm{T}: \\
23 \%\end{array}$ & & & \\
\hline & & & & $\begin{array}{l}\text { > Confirmed C/T: } \\
77 \%\end{array}$ & & & \\
\hline & & & & $\begin{array}{l}\text { > Duration med.: } \\
10 \text { days }\end{array}$ & & & \\
\hline 2016 studies & & & & & & & \\
\hline $\begin{array}{l}\text { Conference } \\
\text { proceedings }\end{array}$ & & & & & & & \\
\hline lovleva et al. & 2 & Imipenem-resistant PsA HCAP. & $>\mathrm{APACHE}$ & - & 100 & 100 & 0 \\
\hline & & & 13 & & $(2 / 2)$ & $(2 / 2)$ & \\
\hline $\begin{array}{l}\text { single center, } \\
\text { case series }\end{array}$ & & & $\begin{array}{l}>\mathrm{CCl} \\
\text { mean }=2\end{array}$ & & & & \\
\hline US & & & & & & & \\
\hline $\begin{array}{l}\text { Nathan et al. } \\
\text { 2016(99) }\end{array}$ & 28 & $\begin{array}{l}\text { Gram-negative infections ( } 68 \% \\
\text { resistant pathogens, including }\end{array}$ & $>\operatorname{ICUN}=0$ & $\begin{array}{l}\text { > Duration: } \\
\text { med }=12 \text { davs }\end{array}$ & 89 & - & - \\
\hline & & $36.4 \%$ MDR PsA and $15.2 \%$ ESBL- & & for RTI, 12 days & $(24 / 27)$ & & \\
\hline $\begin{array}{l}\text { Retrospective, } \\
\text { multicenter }\end{array}$ & & $\begin{array}{l}\text { producing E. coli), including RTI } \\
(28.6 \%) \text {, cIAI }(25 \%) \text {, and cUTI (25\%). }\end{array}$ & & $\begin{array}{l}\text { for ClAl and } 15 \\
\text { days for cUTI }\end{array}$ & & & \\
\hline US & & & & & & & \\
\hline 2015 studies & & & & & & & \\
\hline $\begin{array}{l}\text { Peer-reviewed } \\
\text { literature }\end{array}$ & & & & & & & \\
\hline
\end{tabular}

Page 13/28 


\begin{tabular}{|c|c|c|c|c|c|c|c|}
\hline \multirow{2}{*}{$\begin{array}{l}\text { Citation, } \\
\text { study design, } \\
\text { location }\end{array}$} & \multirow{2}{*}{$\begin{array}{l}\mathrm{N} \\
\mathrm{C} / \mathrm{T}\end{array}$} & \multirow[t]{2}{*}{ Patient/infection description } & \multirow{2}{*}{$\begin{array}{l}\text { Disease } \\
\text { severity }\end{array}$} & \multirow[t]{2}{*}{$\mathrm{C} / \mathrm{T}$ treatment } & \multicolumn{3}{|c|}{ Outcome, \% (n/N) } \\
\hline & & & & & Clinical & Micro. & Mortality \\
\hline $\begin{array}{l}\text { Gelfand et al. } \\
2015(43)\end{array}$ & \multirow[t]{2}{*}{3} & \multirow[t]{2}{*}{ MDR PsA pneumonia. } & \multirow[t]{2}{*}{$>\mathrm{IMC}=2$} & $\begin{array}{l}\text { > Dose C/T: } 3 \mathrm{~g} \\
\text { q8h }(100 \%)\end{array}$ & & & 0 \\
\hline $\begin{array}{l}\text { Retrospective, } \\
\text { single center, } \\
\text { case series }\end{array}$ & & & & $\begin{array}{l}\text { > Duration mean } \\
\text { (range): } 12.7 \\
(10-14) \text { days }\end{array}$ & $(3 / 3)$ & $(3 / 3)$ & \\
\hline US & & & & & & & \\
\hline
\end{tabular}

${ }^{a} 2$ patients died - both completed therapy and were in the clinical cure group, but later succumbed to comorbid conditions.

${ }^{b} 2$ of the 7 courses were considered clinical failures. One patient with clinical failure then had a successful $C / T$ course.

${ }^{\mathrm{C}}$ This study contains a subset of patients identified in Puzniak et al. 2018.(87)

${ }^{\mathrm{d}}$ Although all patients had a favorable clinical outcome, 4 patients were reported to have died from other causes.

eMedian duration of therapy in patients who received pathogen-directed therapy was 8 days; empiric-turned-pathogen-directed therapy, 8 days; empiric-remained-empiric therapy, 7.5 days; and empiric therapy that was subsequently changed or discontinued, 1 day.

fOnly assessed in patients with C/T-susceptible infections.

ABSSSI: Acute bacterial skin and skin structure infection; APACHE: Acute Physiology and Chronic Health Evaluation; BSI: Bloodstream infection; CCl: Charlson Comorbidity index; Cl: Continuous infusion; clAl: Complicated intra-abdominal infection; CF: Cystic fibrosis; CLABSI: Central-line-associated bloodstream infection; CR: Carbapenem-resistant; CSSTI: Complicated skin and soft tissue infection; C/T: Ceftolozane/tazobactam; cUTI: Complicated urinary tract infection; ESBL: Extended-spectrum $\beta$-lactamase; HABP: Hospitalacquired bacterial pneumonia; HCAP: Healthcare-associated pneumonia; IAl: Intra-abdominal infection; ICU: Intensive care unit; IMC: Immunocompromised; IQR: Interquartile range; LVAD: Left-ventricular assist device; MDR: Multidrug-resistant; NP: Nosocomial pneumonia; NR: Not reported; PDR: Pandrug-resistant; PSA: Pseudomonas aeruginosa; RTI: Respiratory tract infection; SD: Standard deviation; SOFA: Sequential Organ Failure Assessment; SSTI: Skin and soft tissue infection; US: United States; UTI: Urinary tract infection; VABP: Ventilator-associated bacterial pneumonia; XDR: Extensively-drug-resistant.

\subsection{Patient characteristics}

Identified studies included a total 3,701 distinct patients treated with $\mathrm{C} / \mathrm{T}$. Excluding the single-patient case reports, the median number of patients included was 30 (range: 2(100)-1,490(87)). Patient populations were heterogeneous, with a number of different sources of infections and pathogens reported. There were 3,735 total infections. Of these, there were 1,807 infections where the source of infection was not reported (48.4\%); excluding those publications, the most common source of infection(s) were pneumonia/respiratory tract infections (RTIs; $52.9 \%$ of reported infections), UTIs (14.9\%), and IAls (10.1\%). There was also report of C/T use in SSTIs (7.1\%), bone and joint infections (6.1\%), and primary bacteremia (4.2\%). Over time, the number of patients treated with $\mathrm{C} / \mathrm{T}$ has grown, but the proportion of each infection type has remained relatively consistent (Fig. 2). The number of patients treated for RTIs was consistently high over the time period studied (Fig. 2) - 100.0\% of identified patients treated with C/T in 2015, 35.3\% in 2016, 65.5\% in 2017, $44.9 \%$ in $2018,62.9 \%$ in 2019 , and $49.1 \%$ in 2020 had RT Is, and the number of patients treated with C/T for these infections has grown year-on-year.

The patient population included in these RWE publications were often classified as seriously ill with multiple comorbidities. In total, 1,751 patients ( $47.3 \%$ of 3,701 patients reported) were admitted to the ICU. The literature review recorded three commonly used measures of patient illness severity - Acute Physiology and Chronic Health Evaluation (APACHE) II, Sequential Organ Failure Assessment (SOFA), and Charlson Comorbidity (CC) index. APACHE and SOFA are systems for predicting ICU mortality. Nine publications, comprising 794 patients treated with $\mathrm{C} / \mathrm{T}$, reported APACHE scores ranging from 13-40, with larger studies (> 50 patients) ranging from $18-40 .(22,24,33,36,80,84$, $90,97,100)$ Six publications, comprising 472 patients treated with C/T, reported SOFA scores ranging from $3-8 .(22,26,27,30,38,39)$ The CC index quantifies the comorbidity burden of included patients by predicting the mortality of patients with multiple comorbidities. Twentyone publications, comprising 2,930 patients, reported CC index scores ranging from 2-6.(18, 22, 24-28, 30-33, 39, 40, 80, 84, 86, 87, 90, 96, $97,100)$ These measures show the high severity of illness of patients included in the RWE of C/T treatment.

Furthermore, this review identified 30 publications reporting a total of 364 immunocompromised patients. $(22,26,27,30,34,37-39,41,43$, $48,49,51,53,59-61,63,68,73,79,83-86,90,91,96-98)$ Immunocompromised patients include those with a history of organ transplant, disease suppressing immunity (e.g. human immunodeficiency virus [HIV]/acquired immunodeficiency syndrome [AIDS], Iymphoma, 
leukemia), receipt of chemotherapy, or immunosuppressive treatment (e.g. corticosteroids). Of these studies, 5 reported only immunocompromised patients. $(26,34,43,79,84)$

A total of 1,294 (35.0\%) patients did not have a causative pathogen specified (note that the majority of these came from a single publication (Puzniak et al. 2018).(87) For publications that reported a causative pathogen, the majority of patients $(90.7 \%$; $N=2,184)$ had infections that were caused by $P$. aeruginosa, of which $14.0 \%$ were caused by non-drug resistant $P$. aeruginosa, or the level of resistance was not specified, $72.3 \%$ by MDR P. aeruginosa, $13.4 \%$ extensively-drug-resistant (XDR), and $0.2 \%$ pan-drug-resistant (PDR). Note that the level of resistance specified (MDR/XDR/PDR) was recorded as described in the publication. Resistant infections comprised the majority of infections treated in studies published in the first three-year period captured (2015-2017) vs. the second three-year period (Fig. 3).

\subsection{Treatment characteristics}

$\mathrm{C} / \mathrm{T}$ is indicated for use at two doses: either $1.5 \mathrm{~g}$ q8h (for clAl and $\mathrm{cUTI}$ ) or $3 \mathrm{~g}$ q8h (for patients with HABP/VABP). For patients with renal insufficiency, doses are reduced according to level of creatinine clearance. In studies that reported dosing information ( $\mathrm{N}=1,418$ patients), $\mathrm{C} / \mathrm{T}$ was used as a $1.5 \mathrm{~g}$ q8h dose in 619 (43.7\%) patients, as a $3 \mathrm{~g}$ q8h dose in 621 (43.8\%) patients, and as a creatinine clearance adjusted dose in 178 (12.6\%) patients. Note, however, that reporting of dosing was inconsistent between studies and the specific dose by type of infection (i.e., $3 \mathrm{~g}$ q8h for respiratory) was not always delineated. Of studies that reported the timing of $\mathrm{C} / \mathrm{T}$ treatment ( $\mathrm{N}=893 \mathrm{patients})$, $\mathrm{C} / \mathrm{T}$ was administered empirically (i.e. prior to susceptibility results) in 222 (24.9\%) patients and administered confirmed (i.e. following susceptibility results) in 671 (75.1\%). There was little year-on-year change in the proportion of patients treated empirically or confirmed, or treated with a $1.5 \mathrm{~g}$ q8h or $3 \mathrm{~g}$ q8h regimen - despite the approval of the $3 \mathrm{~g}$ q8h dose in 2019.

There was large variation in the duration of C/T therapy reported, often different to the label dose of 4-14 (clAl), 7 (cUTI), or 8-14 (HABP/VABP) days. In all studies, the median duration of $\mathrm{C} / \mathrm{T}$ therapy ranged from 7-56 days, irrespective of dose. Median duration in larger studies (> 50 patients) ranged from 8-16.1 days, consistent with the indicated duration.

\subsection{Outcomes}

\subsubsection{Overall outcomes}

All 47 studies that included more than one patient reported clinical outcomes with C/T treatment: 39 reported clinical outcomes,(18-21, 23$43,81,83-86,90,92,94-100) 19$ reported microbiological outcomes,(19, 21, 24, 31-33, 35-38, 40, 41, 43, 80, 94, 96-98, 100) and 45 reported mortality rates.(18-43, 79, 80, 82-92, 94-98, 100) Clinical success rates ranged from 45.7-100.0\%, with 27 studies (69\%) reporting clinical success rates of $>70 \%$. In larger studies (> 50 patients; 10 studies), clinical success rates ranged from $56.7-83.7 \%$. Microbiological success rates were similar, ranging from $31-100 \%$, with 14 studies (74\%) reporting microbiological success rates of $>70 \%$. In larger studies (> 50 patients; three studies), microbiological success rates ranged from 31-75.3\%. Mortality rates ranged from 0-50\%, with 31 studies (69\%) reporting mortality rates of $\leq 20 \%$. In larger studies (> 50 patients; 16 studies), mortality rates ranged from 5-29\%. With each of these outcomes, note that definitions used, and assessments performed, were variable.

Outcomes were consistent in the 36 single-patient case reports - clinical cure was reported in 28 of 32 studies (87.5\%), microbiological cure in 18 of 23 studies (78.3\%), and mortality in 4 of 32 studies $(11.4 \%)$.

\subsubsection{Outcomes by treatment characteristics}

Seven studies reported on the treatment characteristics that were risk factors for clinical outcomes. $(18,25,28,30,32,33,39)$ Patient cohort size ranged from $21-205$, with a median of 90 . Five studies included patients with $P$. aeruginosa infections; $(25,28,30,32,33,39)$ one included patients with Enterobacterales infections.(18) There was a diverse range of infection types included.

Five studies found mixed evidence that a delay in receipt of $\mathrm{C} / \mathrm{T}$ led to worse outcomes. $(18,28,30,33,39) \mathrm{Bassetti}$ et al. 2020 found that a significantly higher proportion of patients who achieved clinical success received empiric $\mathrm{C} / \mathrm{T}$ and had a significantly shorter latency between infection onset and C/T administration (both $\mathrm{p}<0.001)$.(18) Similarly, Gallagher et al. 2018 found that starting $\mathrm{C} / \mathrm{T}$ less than four days after positive culture was associated with significantly higher clinical and microbiological cure rates, and that starting $\mathrm{C} / \mathrm{T}$ more than four days after positive culture was associated with significantly higher mortality.(33) In contrast, three studies found no association between initiating $\mathrm{C} / \mathrm{T}$ within 48 hours of $P$. aeruginosa isolation, time to $\mathrm{C} / \mathrm{T}$, or type of treatment (empiric, semi-empiric, or confirmed) (all $\mathrm{p}$ $>0.05)$. $(28,30,39)$ These three studies were of smaller size (169 combined patients vs. 258 for the two previously mentioned studies), and, importantly, Rodriguez-Nunez et al. included some patients that were also reported in Diaz-Cañestro et al. 2018, effectively double-counting these patients and possibly giving them disproportionate influence over the conclusion drawn in this review. $(28,30)$

\subsubsection{Outcomes by PsA resistance subtype}

Page 15/28 
Two studies were identified that conducted an analysis to understand whether $P$. aeruginosa resistance was a factor in clinical outcome.(28, 30) In univariate analysis, Rodriguez-Nunez et al. found that similar proportions of survivors and non-survivors had XDR PsA infections.(28) Whereas, Diaz-Cañestro et al. found that resistance profile (the proportion of patients with MDR vs. XDR infections) was significantly different between patients who were clinical successes or failures (Table 3).(30)

Table 3

PsA resistance risk factors for clinical outcomes

\begin{tabular}{|c|c|c|c|c|c|c|c|}
\hline \multirow{2}{*}{$\begin{array}{l}\text { Citation, study } \\
\text { design, } \\
\text { location } \\
\text { Rodriguez- } \\
\text { Nunez et al. } \\
2019(28)\end{array}$} & \multirow{3}{*}{$\begin{array}{l}\mathbf{N} \\
\mathrm{C} / \mathrm{T}\end{array}$} & \multirow{3}{*}{$\begin{array}{l}\text { Patient/infection description } \\
\text { Drug-resistant PSA RTIS (76.7\% XDR; } 23.3 \% \\
\text { MDR). }\end{array}$} & \multirow{3}{*}{$\begin{array}{l}\text { Analysis } \\
\text { Univariate } \\
\text { regression }\end{array}$} & \multirow{3}{*}{$\begin{array}{l}\text { Variable } \\
\begin{array}{l}\text { XDR PsA } \\
\text { infection }\end{array}\end{array}$} & \multicolumn{2}{|c|}{$\begin{array}{l}\text { Proportion of patients } \\
\text { with either outcome with } \\
\text { variable }\end{array}$} & \multirow[t]{2}{*}{$\begin{array}{l}\text { p- } \\
\text { value }\end{array}$} \\
\hline & & & & & $\begin{array}{l}\text { Survivors } \\
(N=65)\end{array}$ & $\begin{array}{l}\text { Non- } \\
\text { survivors } \\
(N=25)\end{array}$ & \\
\hline $\begin{array}{l}\text { multicenter } \\
\text { International }\end{array}$ & & & & & $\begin{array}{l}73.8 \% \\
(48 / 65)\end{array}$ & $\begin{array}{l}84.0 \% \\
(21 / 25)\end{array}$ & .308 \\
\hline \multirow{4}{*}{$\begin{array}{l}\text { Diaz-Cañestro } \\
\text { et al. 2018(30) } \\
\text { Prospective, } \\
\text { single center } \\
\text { Spain }\end{array}$} & \multirow[t]{4}{*}{58} & \multirow[t]{4}{*}{$\begin{array}{l}\text { PsA ( } 86.2 \% \text { XDR; } 10.3 \% \text { MDR) infections, } \\
\text { including RTIs }(60.3 \%) \text {, UTIs }(17.2 \%) \text {, and } \\
\text { IAls }(6.9 \%) \text {. }\end{array}$} & \multirow[t]{4}{*}{$\begin{array}{l}\text { Univariate } \\
\text { regression }\end{array}$} & & $\begin{array}{l}\text { Clinical } \\
\text { cure } \\
(N=35)\end{array}$ & $\begin{array}{l}\text { Clinical } \\
\text { failure }(N= \\
21)\end{array}$ & \\
\hline & & & & $\begin{array}{l}\text { Resistance } \\
\text { profile }\end{array}$ & & & .045 \\
\hline & & & & $\begin{array}{l}\text { XDR PsA } \\
\text { infection }\end{array}$ & $\begin{array}{l}82.8 \% \\
(29 / 35)\end{array}$ & $\begin{array}{l}100.0 \% \\
(21 / 21)\end{array}$ & \\
\hline & & & & $\begin{array}{l}\text { MDR PsA } \\
\text { infection }\end{array}$ & $\begin{array}{l}17.1 \% \\
(6 / 35)\end{array}$ & $\begin{array}{l}0.0 \% \\
(0 / 21)\end{array}$ & \\
\hline
\end{tabular}

\subsection{Comparative studies}

Five studies were identified that compared $\mathrm{C} / \mathrm{T}$ with other treatment regimens (Table 4): three included aminoglycoside/polymyxin-based regimens as comparator; $(23,27,80)$ two either used standard of care $(\mathrm{SoC}) .(26,83)$ Each study included patients with $P$. aeruginosa infections, with four including patients with resistant $P$. aeruginosa. $(23,27,80,83)$

In the three studies with aminoglycoside-/polymyxin-based comparators, all reported mortality rates, $(23,27,80)$ two reported clinical cure rates, $(23,27)$ and one reported microbiological cure rate.(80) In Pogue et al., patients treated with $\mathrm{C} / \mathrm{T}$ had significantly higher clinical cure rate $(p=0.002)$, but there was no difference in in-hospital mortality.(27) In response, Vena et al. conducted a similar case-control study, but balanced the proportion of patients with pneumonia in each arm, ensured patients received a sufficient polymyxin dosage, and ensured that all included patients had an infectious disease consultation.(23) Results were comparable with Pogue et al. - patients treated with C/T had a numerically higher clinical cure rate and lower mortality rate than patients treated with aminoglycoside/polymyxin regimen, though this did not reach statistical significance.(23) Caffrey et al. showed that patients treated with $\mathrm{C} / \mathrm{T}$ were significantly less likely to die as inpatients than patients treated with aminoglycoside/polymyxin-based regimens, although there was no difference in 30-day mortality rates or microbiological cure rates, and clinical cure rates were not reported.(80)

In the two studies that compared patients treated with $\mathrm{C} / \mathrm{T}$ with mixed SoC antibacterial agents, both reported clinical cure rates and mortality. $(26,83)$ Both studies found that patients treated with $\mathrm{C} / \mathrm{T}$ had numerically higher clinical cure rates than patients treated with other antibacterial agents. Fernández-Cruz et al. additionally found that patients treated with $\mathrm{C} / \mathrm{T}$ had significantly lower mortality rates ( $\mathrm{p}<0.05$ ); (26) such a difference was not apparent in Mills et al.(83) 
Table 4

Studies reporting comparative data

\begin{tabular}{|c|c|c|c|c|c|c|c|}
\hline \multirow{2}{*}{$\begin{array}{l}\text { Citation, } \\
\text { study design, } \\
\text { location }\end{array}$} & \multirow{2}{*}{$\begin{array}{l}\text { Study } \\
\text { design }\end{array}$} & \multirow{2}{*}{$\begin{array}{l}\text { Patient/infection } \\
\text { description }\end{array}$} & \multirow[t]{2}{*}{ Treatment groups } & \multirow{2}{*}{$\begin{array}{l}\text { Outcome } \\
\text { description }\end{array}$} & \multicolumn{2}{|c|}{ Outcome, \% (n/N) } & \multirow{2}{*}{$\begin{array}{l}\text { p- } \\
\text { value/aOR }\end{array}$} \\
\hline & & & & & $\mathrm{C} / \mathrm{T}$ & Comparator & \\
\hline \multicolumn{8}{|c|}{ Aminoglycoside/polymyxin comparator } \\
\hline $\begin{array}{l}\text { Caffrey et al. } \\
2020(80)\end{array}$ & \multirow[t]{6}{*}{ Cohort } & \multirow{6}{*}{$\begin{array}{l}\text { Patients had } \\
\text { MDR PsA } \\
\text { infections. }\end{array}$} & \multirow{6}{*}{$\begin{array}{l}\mathrm{C} / \mathrm{T}(\mathrm{N}=57) \text { vs. } \\
\text { aminoglycoside/polymyxin- } \\
\text { based }(\mathrm{N}=155)\end{array}$} & Clinical cure & - & - & - \\
\hline $\begin{array}{l}\text { Retrospective, } \\
\text { multicenter, } \\
\text { cohort }\end{array}$ & & & & $\begin{array}{l}\text { Mortality, 30- } \\
\text { day }\end{array}$ & $\begin{array}{l}17.5 \\
(10 / 57)\end{array}$ & $\begin{array}{l}18.1 \\
(28 / 155)\end{array}$ & $\begin{array}{l}\text { aOR: } 0.78 \\
95 \% \mathrm{Cl}: \\
0.30-2.03\end{array}$ \\
\hline \multirow[t]{4}{*}{ US } & & & & $\begin{array}{l}\text { Mortality, } \\
\text { inpatient }\end{array}$ & $\begin{array}{l}15.8 \\
(9 / 57)\end{array}$ & $\begin{array}{l}27.7 \\
(43 / 155)\end{array}$ & \\
\hline & & & & & & & $\begin{array}{l}95 \% \mathrm{Cl}: \\
0.16-0.93\end{array}$ \\
\hline & & & & $\begin{array}{l}\text { Microbiological } \\
\text { cure }\end{array}$ & $\begin{array}{l}31.0 \\
(13 / 42)\end{array}$ & $\begin{array}{l}30.6 \\
(33 / 108)\end{array}$ & \\
\hline & & & & & & & $\begin{array}{l}95 \% \mathrm{Cl}: \\
0.35-2.21\end{array}$ \\
\hline $\begin{array}{l}\text { Vena et al. } \\
2020(23)\end{array}$ & \multirow[t]{3}{*}{$\begin{array}{l}\text { Case- } \\
\text { control }\end{array}$} & \multirow{3}{*}{$\begin{array}{l}\text { Patients had } \\
\text { pneumonia or } \\
\text { bacteremia } \\
\text { caused by MDR } \\
\text { or XDR PsA. }\end{array}$} & \multirow[t]{3}{*}{$\begin{array}{l}\mathrm{C} / \mathrm{T}(\mathrm{N}=16) \text { vs. } \\
\text { aminoglycoside/polymyxin- } \\
\text { based }(\mathrm{N}=32)\end{array}$} & Clinical cure & $\begin{array}{l}81.3 \\
(13 / 16)\end{array}$ & $\begin{array}{l}56.3 \\
(18 / 32)\end{array}$ & 0.11 \\
\hline \multirow{2}{*}{$\begin{array}{l}\text { Retrospective, } \\
\text { multicenter, } \\
\text { case-control } \\
\text { Italy }\end{array}$} & & & & $\begin{array}{l}\text { Mortality, 30- } \\
\text { day }\end{array}$ & $\begin{array}{l}18.8 \\
(3 / 16)\end{array}$ & $28.1(9 / 32)$ & 0.72 \\
\hline & & & & $\begin{array}{l}\text { Microbiological } \\
\text { cure }\end{array}$ & - & - & - \\
\hline $\begin{array}{l}\text { Pogue et al. } \\
2019(27)\end{array}$ & \multirow[t]{3}{*}{$\begin{array}{l}\text { Case- } \\
\text { control }\end{array}$} & \multirow[t]{3}{*}{$\begin{array}{l}\text { Patients had an } \\
\text { MDR or XDR } \\
\text { PsA infection. }\end{array}$} & \multirow[t]{3}{*}{$\begin{array}{l}\mathrm{C} / \mathrm{T}(\mathrm{N}=100) \text { vs. } \\
\text { aminoglycoside/polymyxin- } \\
\text { based }(\mathrm{N}=100)\end{array}$} & Clinical cure & $\begin{array}{l}81.0 \\
(81 / 100)\end{array}$ & $\begin{array}{l}61.0 \\
(61 / 100)\end{array}$ & 0.002 \\
\hline \multirow{2}{*}{$\begin{array}{l}\text { Retrospective, } \\
\text { multicenter, } \\
\text { case-control } \\
\text { US }\end{array}$} & & & & $\begin{array}{l}\text { Mortality, in } \\
\text { hospital }\end{array}$ & $\begin{array}{l}20.0 \\
(20 / 100)\end{array}$ & $\begin{array}{l}25.0 \\
(25 / 100)\end{array}$ & 0.400 \\
\hline & & & & $\begin{array}{l}\text { Microbiological } \\
\text { cure }\end{array}$ & - & - & - \\
\hline \multicolumn{8}{|c|}{ Other comparator } \\
\hline $\begin{array}{l}\text { Fernández- } \\
\text { Cruz et al. } \\
2019(26)\end{array}$ & $\begin{array}{l}\text { Case- } \\
\text { control }\end{array}$ & $\begin{array}{l}\text { Patients had } \\
\text { hematological } \\
\text { malignancies }\end{array}$ & $\begin{array}{l}\mathrm{C} / \mathrm{T}(\mathrm{N}=19) \text { vs. mixed SoC } \\
\text { antibacterial agents }(\mathrm{N}= \\
38)\end{array}$ & $\begin{array}{l}\text { Clinical cure, } \\
\text { 14-day }\end{array}$ & $\begin{array}{l}89.5 \\
(17 / 19)\end{array}$ & $\begin{array}{l}71.1 \\
(27 / 38)\end{array}$ & 0.183 \\
\hline \multirow{2}{*}{$\begin{array}{l}\text { Retrospective, } \\
\text { single center, } \\
\text { case-control } \\
\text { Spain }\end{array}$} & & $\begin{array}{l}\text { and PsA } \\
\text { infection. }\end{array}$ & & $\begin{array}{l}\text { Mortality, 30- } \\
\text { day }\end{array}$ & $\begin{array}{l}5.3 \\
(1 / 19)\end{array}$ & $\begin{array}{l}28.9 \\
(11 / 38)\end{array}$ & 0.045 \\
\hline & & & & $\begin{array}{l}\text { Microbiological } \\
\text { cure }\end{array}$ & - & - & - \\
\hline $\begin{array}{l}\text { Mills et al. } \\
2019(83)\end{array}$ & Cohort & $\begin{array}{l}\text { Patients had } \\
\text { pneumonia with } \\
\text { an MDR PsA }\end{array}$ & $\begin{array}{l}\mathrm{C} / \mathrm{T}(\mathrm{N}=62) \text { vs. mixed SoC } \\
\text { antibacterial agents }(\mathrm{N}= \\
53)\end{array}$ & $\begin{array}{l}\text { Clinical cure, } \\
\text { 14-day }\end{array}$ & $\begin{array}{l}72.6 \\
(45 / 62)\end{array}$ & $\begin{array}{l}67.9 \\
(36 / 53)\end{array}$ & 0.683 \\
\hline \multirow{2}{*}{$\begin{array}{l}\text { Retrospective, } \\
\text { multicenter } \\
\text { cohort } \\
\text { US }\end{array}$} & & culture. & & Mortality & $\begin{array}{l}29.0 \\
(18 / 62)\end{array}$ & $\begin{array}{l}26.4 \\
(14 / 53)\end{array}$ & 0.840 \\
\hline & & & & $\begin{array}{l}\text { Microbiological } \\
\text { cure }\end{array}$ & - & - & - \\
\hline
\end{tabular}

\section{Discussion}

The principal finding of this SLR was that there is a body of RWE that establishes the effectiveness of C/T in real-world clinical practice, including patients described as severely ill patients and/or with resistant infections. Considering the patient disease severity measures, publications reported APACHE scores ranging from 13-40, with larger studies (> 50 patients) ranging from 18-40.(22, 24, 33, 36, 80, 84, 90, $97,100)$ This is higher than the APACHE score reported in ASPECT-NP (median 17),(15) and significantly higher than reported in ASPECT-IAI 
(mean 6.2).(16) Furthermore, inclusion of immunocompromised patients, typically excluded by clinical trials, offers valuable insights into $\mathrm{C} / \mathrm{T}$ effectiveness in this underrepresented population. A key limitation of many clinical trials is the exclusion of these seriously ill patients, and the restriction of recruitment to only patients with a narrow range of infections. By filling this gap, the RWE therefore provides valuable data on the outcomes of these patients seen in clinical practice.

Despite the heterogeneity in the patient population, outcomes of treatment with $\mathrm{C} / \mathrm{T}$ were consistent with those found in the ASPECT clinical trials. In larger RWE studies (> 50 patients), clinical cure rates ranged from $56.7-83.7 \%$, microbiological cure rates ranged from $31-75.3 \%$, and mortality rates ranged from $5-29 \%$. By way of descriptive comparison, C/T outcomes in the ASPECT trials were: ASPECT-cUTI, clinical cure $=92.0 \%$, microbiological eradication $=80.4 \%$, and mortality $=0.2 \% ;(17,101)$ ASPECT-clAl, clinical cure $=83.0 \%$, microbiological cure $=$ $85.3 \%$, and mortality $=2.3 \% ;(16,102)$ and ASPECT-NP, clinical cure $=54.4 \%$, microbiological eradication $=73.1 \%$, and 28 -day mortality $=$ 24.0\%.(15)

Treatment characteristics were broadly aligned with the approved use of $\mathrm{C} / \mathrm{T}$ and both indicated doses of $\mathrm{C} / \mathrm{T}$ were used approximately equally; however, it was unclear which dose was used for which indication and often the outcomes were not stratified by dose and indication. This result is concerning, since the indicated dose for pneumonia is based on optimized pharmacokinetic and pharmacodynamic properties. C/T was more commonly used as confirmed therapy than as an empiric therapy (75.1\% vs. $24.9 \%)$. This is consistent with the principles of antimicrobial stewardship, whereby broader-spectrum antibacterial agents are reserved for special clinical situations when other treatments have failed. However, there were two studies that suggested patients who were treated earlier; either empirically, or sooner after infection onset, had better clinical outcomes. $(18,33)$ Although a similar association was not found in three other studies,(28, 30, 39) comparison of early vs. late use of $\mathrm{C} / \mathrm{T}$ warrants further investigation. Late use of $\mathrm{C} / \mathrm{T}$ may be indicative of initial inappropriate antibacterial therapy with other agents, which has been shown in the literature to have deleterious effects on outcomes. $(8,9)$

Data from the comparative studies suggest that $\mathrm{C} / \mathrm{T}$ is at least as effective as, and in several cases, significantly better than, aminoglycoside- or polymyxin-based regimens for serious, MDR infections. $(23,27,80)$ Outside the scope of this review, though pertinent to clinicians, is the lower risk of nephrotoxicity with $\mathrm{C} / \mathrm{T}$ compared to aminoglycosides or polymyxins. Both comparative studies that assessed safety found a significantly lower incidence of acute kidney injury with $\mathrm{C} / \mathrm{T}$ than with aminoglycoside/polymyxin-based comparators.(23, 27) This combination of comparable effectiveness and lower risk of nephrotoxicity means that $\mathrm{C} / \mathrm{T}$ can be an alternative to these therapies, particularly in patients with decreased renal function.

This SLR highlights the inconsistent reporting that is common within published RWE. Due to differences in study design, objectives, outcome assessment and definitions, there were often incomplete data for the variables of interest, as set out in this SLR. This variability in turn imposes challenges in attributing outcomes to the exposure studied. The inclusion of conference proceedings, which are not subject to the same rigorous peer-review, may have affected evidence included within this review, and thus the conclusions drawn. As mentioned in the results, some studies included data that were reported in part by other studies - this may be more widespread than thought as some large database studies collected patients across hundreds of hospitals, possibly capturing patients reported in other studies. As this is a qualitative review, this double counting was not adjusted for. However, given the consistency of outcomes between studies conducted in different locations, in different years, and by different authors, it is likely that the outcomes reported approximate the true treatment effect.

As was to be expected, many studies had small sample sizes and did not include comparison groups for statistical inference purposes. In the comparative cohort studies that did, C/T had comparable efficacy to standard of care, and was significantly better in several outcomes. Furthermore, identified risk factors may have been subject to a reporting bias: with some studies only reporting multivariate analysis, it was difficult to recognize which risk factors were non-significant, and therefore excluded, in univariate analysis. Many studies had industry authors and/or were sponsored by grants from industry which may lead to publication bias; however, the results were consistent regardless of authorship or sponsorship. The vast majority of publications were of a retrospective design. This may lead to selection bias, as both exposure and outcome of patients are already known. Finally, this review did not include a comprehensive search of all relevant microbiology conferences or search for studies that were not captured in biomedical databases. These are pragmatic limitations associated with all literature reviews and are not expected to influence the findings of this review.

In conclusion, this SLR identified and summarized the published RWE on the use of C/T in clinical practice. These studies demonstrate the clinical effectiveness of $\mathrm{C} / \mathrm{T}$, despite the diverse group of seriously ill patients and level of resistance of the pathogens treated. The RWE body of literature provides additional insights into patient types that are commonly encountered in everyday practice and may have been excluded from the registration trials. Further studies are needed that evaluate homogenous patient sub-types and that account for other treatments that were received prior to $\mathrm{C} / \mathrm{T}$ to properly attribute outcomes to the effectiveness of $\mathrm{C} / \mathrm{T}$.

\section{List Of Abbreviations}




\begin{tabular}{|c|c|}
\hline ABSSSI & Acute bacterial skin and skin structure infection \\
\hline AIDS & Acquired immunodeficiency syndrome \\
\hline $\mathrm{aOR}$ & Adjusted odds ratio \\
\hline BSI & Bloodstream infection \\
\hline $\mathrm{CC}(\mathrm{I})$ & Charlson comorbidity (index) \\
\hline $\mathrm{CF}$ & Cystic Fibrosis \\
\hline $\mathrm{Cl}$ & Confidence interval \\
\hline clAl & Complicated intra-abdominal infection \\
\hline CLABSI & Central-line-associated bloodstream infection \\
\hline CNS & Central nervous system \\
\hline $\mathrm{CR}$ & Carbapenem-resistant \\
\hline cSSTI & Complicated skin and soft tissue infection \\
\hline $\mathrm{C} / \mathrm{T}$ & Ceftolozane/tazobactam \\
\hline cUTI & Complicated urinary tract infection \\
\hline ECCMID & European Congress of Clinical Microbiology and Infectious Diseases \\
\hline ESBL & Extended-spectrum $\beta$-lactamase \\
\hline HABP & Hospital-acquired bacterial pneumonia \\
\hline $\mathrm{HAl}$ & Hospital-acquired infection \\
\hline HCAP & Healthcare-associated pneumonia \\
\hline HIV & Human immunodeficiency syndrome \\
\hline$|A|$ & Intra-abdominal infection \\
\hline ICU & Intensive care unit \\
\hline IDWeek & Infectious Disease Week \\
\hline IMC & Immunocompromised \\
\hline IQR & Interquartile range \\
\hline LOS & Length of stay \\
\hline LVAD & Left-ventricular assist device \\
\hline MDR & Multidrug-resistant \\
\hline NP & Nosocomial pneumonia \\
\hline NR & Not reported \\
\hline OR & Odds ratio \\
\hline PDR & Pandrug-resistant \\
\hline $\mathrm{PK} / \mathrm{PD}$ & Pharmacokinetics/pharmacodynamics \\
\hline PsA & Pseudomonas aeruginosa \\
\hline RCT & Randomized controlled trial \\
\hline RTI & Respiratory tract infection \\
\hline RWE & Real-world evidence \\
\hline SD & Standard deviation \\
\hline SLR & Systematic literature review \\
\hline
\end{tabular}

Page 19/28 


\begin{tabular}{|ll|}
\hline SOC & Standard of care \\
\hline SOFA & Sequential Organ Failure Assessment \\
\hline SSTI & Skin and soft tissue infection \\
\hline UK & United Kingdom \\
\hline US & United States \\
\hline UTI & Urinary tract infection \\
\hline VABP & Ventilator-associated bacterial pneumonia \\
\hline WHO & World Health Organization \\
\hline XDR & Extensively-drug-resistant \\
\hline
\end{tabular}

\section{Declarations}

\subsection{Ethics approval and consent to participate}

Not applicable.

\subsection{Consent for publication}

Not applicable.

\subsection{Availability of data and materials}

All data analyzed during this study are included in this published article (and its supplementary information).

\subsection{Competing interests}

LP and RD are employees of Merck \& Co., Inc.,, who may own stock and/or hold stock options in the Company. TP, HC, and AE are employees of Adelphi Values PROVE, which received funding for this research.

\subsection{Funding}

Funding for this research was provided by Merck \& Co., Inc., Kenilworth, NJ, USA. Employees of the study sponsor were involved in the study design, as well as collection, analysis, and interpretation of the data, and in critically revising the manuscript for important intellectual content.

\subsection{Authors' contributions}

LP and RD conceived and designed the research, contributed to the interpretation of results, and critically revised the manuscript for important intellectual content. TP, HC, and AE conducted the literature review, analyzed the data, interpreted the results, and drafted the manuscript. All authors have approved the manuscript to be submitted for publication.

\subsection{Acknowledgements}

Not applicable.

\section{References}

1. Centers for Disease Control and Prevention. Antibiotic resistant threats in the United States 2019 [Available from: https://www.cdc.gov/drugresistance/pdf/threats-report/2019-ar-threats-report-508.pdf. 
2. Weiner LM WA, Limbago B, et al. Antimicrobial-resistant pathogens associated with healthcare-associated infections: summary of data reported to the National Healthcare Safety Network at the Centers for Disease Control and Prevention, 2011-2014. Infection control \& hospital epidemiology 2016;37(11):1288-301.

3. Peleg AY HD. Hospital-acquired infections due to gram-negative bacteria. The New England journal of medicine. 2010;362(19):1804-13.

4. Ulu AC KB, Inal AS, et al. Risk Factors of Carbapenem-Resistant Klebsiella pneumoniae Infection: A Serious Threat in ICUs. Medical Science Monitor: International Medical Journal of Experimental and Clinical Research. 2015;21:219-24.

5. Infectious Diseases Society of A. Combating Antimicrobial Resistance: Policy Recommendations to Save Lives. Clinical Infectious Diseases. 2011;52(suppl_5):S397-S428.

6. Martin-Loeches I, Torres A, Rinaudo M, Terraneo S, de Rosa F, Ramirez P, et al. Resistance patterns and outcomes in intensive care unit (ICU)-acquired pneumonia. Validation of European Centre for Disease Prevention and Control (ECDC) and the Centers for Disease Control and Prevention (CDC) classification of multidrug resistant organisms. J Infect. 2015;70(3):213-22.

7. Tabak YP, Merchant S, Ye G, Vankeepuram L, Gupta V, Kurtz SG, et al. Incremental clinical and economic burden of suspected respiratory infections due to multi-drug-resistant Pseudomonas aeruginosa in the United States. J Hosp Infect. 2019;103(2):134-41.

8. Bonine NG, Berger A, Altincatal A, Wang R, Bhagnani T, Gillard P, et al. Impact of Delayed Appropriate Antibiotic Therapy on Patient Outcomes by Antibiotic Resistance Status From Serious Gram-negative Bacterial Infections. Am J Med Sci. 2019;357(2):103-10.

9. Lodise TP, Zhao Q, Fahrbach K, Gillard PJ, Martin A. A systematic review of the association between delayed appropriate therapy and mortality among patients hospitalized with infections due to Klebsiella pneumoniae or Escherichia coli: how long is too long? BMC Infect Dis. 2018;18(1):625.

10. Avent ML, Rogers BA, Cheng AC, Paterson DL. Current use of aminoglycosides: indications, pharmacokinetics and monitoring for toxicity. Internal Medicine Journal. 2011;41(6):441-9.

11. Ordooei Javan A, Shokouhi S, Sahraei Z. A review on colistin nephrotoxicity. Eur J Clin Pharmacol. 2015;71(7):801-10.

12. World Health Organization. Global Priority List Of Antibiotic-Resistant Bacteria To Guide Research, Discovery, And Development Of New Antibiotics 2017 [Available from: https://www.who.int/medicines/publications/WHO-PPL-Short_Summary_25Feb-ET_NM_WHO.pdf.

13. Merck \& Co. Ceftolozane/tazobactam (ZERBAXA®) [Prescribing information]. 2019.

14. Merck Sharp \& Dohme. Ceftolozane/tazobactam (Zerbaxa) Summary of Product Characteristics. 2019.

15. Kollef MH, Nováček M, Kivistik Ü, Réa-Neto Á, Shime N, Martin-Loeches I, et al. Ceftolozane-tazobactam versus meropenem for treatment of nosocomial pneumonia (ASPECT-NP): a randomised, controlled, double-blind, phase 3, non-inferiority trial. Lancet Infect Dis. 2019;19(12):1299-311.

16. Solomkin J, Hershberger E, Miller B, Popejoy M, Friedland I, Steenbergen J, et al. Ceftolozane/Tazobactam Plus Metronidazole for Complicated Intra-abdominal Infections in an Era of Multidrug Resistance: Results From a Randomized, Double-Blind, Phase 3 Trial (ASPECT-clAl). Clin Infect Dis. 2015;60(10):1462-71.

17. Wagenlehner FM, Umeh O, Steenbergen J, Yuan G, Darouiche RO. Ceftolozane-tazobactam compared with levofloxacin in the treatment of complicated urinary-tract infections, including pyelonephritis: a randomised, double-blind, phase 3 trial (ASPECT-cUTI). The Lancet. 2015;385(9981):1949-56.

18. Bassetti M, Vena A, Giacobbe DR, Falcone M, Tiseo G, Giannella M, et al. Ceftolozane/Tazobactam for Treatment of Severe ESBLProducing Enterobacterales Infections: A Multicenter Nationwide Clinical Experience (CEFTABUSE II Study). Open Forum Infectious Diseases. 2020;7(5):ofaa139.

19. Bosaeed M, Ahmad A, Alali A, Mahmoud E, Alswidan L, Alsaedy A, et al. Experience With Ceftolozane-Tazobactam for the Treatment of Serious Pseudomonas aeruginosa Infections in Saudi Tertiary Care Center. Infectious Diseases: Research and Treatment. 2020;13(no pagination).

20. Buonomo AR, Maraolo AE, Scotto R, Foggia M, Zappulo E, Congera P, et al. Efficacy and safety of ceftolozane/tazobactam as therapeutic option for complicated skin and soft tissue infections by MDR/XDR Pseudomonas aeruginosa in patients with impaired renal function: a case series from a single-center experience. Infection. 2020;48(2):303-7.

21. Jones BM, Huelfer K, Bland CM. Clinical and safety evaluation of continuously infused ceftolozane/ tazobactam in the outpatient setting. Open Forum Infectious Diseases. 2020;7(2).

22. Jorgensen SCJ, Trinh TD, Zasowski EJ, Lagnf AM, Simon SP, Bhatia S, et al. Real-world experience with ceftolozane-tazobactam for multidrug-resistant gram-negative bacterial infections. Antimicrobial Agents and Chemotherapy. 2020;64(4).

23. Vena A, Giacobbe DR, Mussini C, Cattelan A, Bassetti M, Group ftCS. Clinical Efficacy of Ceftolozane-Tazobactam Versus Other Active Agents for the Treatment of Bacteremia and Nosocomial Pneumonia due to Drug-Resistant Pseudomonas aeruginosa. Clinical Infectious Diseases. 2020.

Page $21 / 28$ 
24. Gerlach AT, Goff DA, Bazan JA. Ceftolozane/Tazobactam for the Treatment of Osteomyelitis Due to Multidrug-Resistant Pseudomonas aeruginosa. Infectious Diseases in Clinical Practice. 2019;27(6):339-42.

25. Bassetti M, Castaldo N, Cattelan A, Mussini C, Righi E, Tascini C, et al. Ceftolozane/tazobactam for the treatment of serious Pseudomonas aeruginosa infections: a multicentre nationwide clinical experience. International Journal of Antimicrobial Agents. 2019;53(4):408-15.

26. Fernandez-Cruz A, Alba N, Semiglia-Chong MA, Padilla B, Rodriguez-Macias G, Kwon M, et al. A case-control study of real-life experience with ceftolozane-tazobactam in patients with hematologic malignancy and pseudomonas aeruginosa infection. Antimicrobial Agents and Chemotherapy. 2019;63 (2) (no pagination)(e02340-18).

27. Pogue JM, Kaye KS, Veve MP, Patel TS, Gerlach AT, Davis SL, et al. Ceftolozane/Tazobactam vs Polymyxin or Aminoglycoside-based Regimens for the Treatment of Drug-resistant Pseudomonas Aeruginosa. Clinical infectious diseases : an official publication of the Infectious Diseases Society of America. 2019;23.

28. Rodriguez-Nunez O, Perianez-Parraga L, Oliver A, Munita JM, Bote A, Gasch O, et al. Higher MICs (>2 mg/L) Predict 30-Day Mortality in Patients With Lower Respiratory Tract Infections Caused by Multidrug- and Extensively Drug-Resistant Pseudomonas aeruginosa Treated With Ceftolozane/Tazobactam. Open Forum Infectious Diseases. 2019;6(10):ofz416.

29. Tan X, Moenster RP. Ceftolozane-tazobactam for the treatment of osteomyelitis caused by multidrug-resistant pathogens: a case series. Therapeutic Advances in Drug Safety. 2019;11(no pagination).

30. Diaz-Canestro M, Perianez L, Mulet X, Martin-Pena ML, Fraile-Ribot PA, Ayestaran I, et al. Ceftolozane/tazobactam for the treatment of multidrug resistant Pseudomonas aeruginosa: experience from the Balearic Islands. European Journal of Clinical Microbiology and Infectious Diseases. 2018;37(11):2191-200.

31. Dietl B, Sanchez I, Arcenillas P, Cuchi E, Gomez L, Gonzalez de Molina FJ, et al. Ceftolozane/tazobactam in the treatment of osteomyelitis and skin and soft-tissue infections due to extensively drug-resistant Pseudomonas aeruginosa: clinical and microbiological outcomes. International Journal of Antimicrobial Agents. 2018;51(3):498-502.

32. Escola-Verge L, Pigrau C, Los-Arcos I, Arevalo A, Vinado B, Campany D, et al. Ceftolozane/tazobactam for the treatment of XDR Pseudomonas aeruginosa infections. Infection. 2018;46(4):461-8.

33. Gallagher JC, Satlin MJ, Elabor A, Saraiya N, McCreary EK, Molnar E, et al. Ceftolozane-Tazobactam for the Treatment of MultidrugResistant Pseudomonas aeruginosa Infections: A Multicenter Study. Open Forum Infectious Diseases. 2018;5(11):ofy280.

34. Hakki M, Lewis JS. Ceftolozane-tazobactam therapy for multidrug-resistant Pseudomonas aeruginosa infections in patients with hematologic malignancies and hematopoietic-cell transplant recipients. Infection. 2018;46(3):431-4.

35. Xipell M, Paredes S, Fresco L, Bodro M, Marco F, Martinez JA, et al. Clinical experience with ceftolozane/tazobactam in patients with serious infections due to resistant Pseudomonas aeruginosa. Journal of Global Antimicrobial Resistance. 2018;13:165-70.

36. Alvarez Lerma F, Munoz Bermudez R, Grau S, Gracia Arnillas MP, Sorli L, Recasens L, et al. Ceftolozane-tazobactam for the treatment of ventilator-associated infections by colistin-resistant Pseudomonas aeruginosa. Revista Espanola de Quimioterapia. 2017;30(3):224-8.

37. Caston JJ, De La Torre A, Ruiz-Camps I, Sorli ML, Torres V, Torre-Cisneros J. Salvage therapy with ceftolozane-tazobactam for multidrug-resistant Pseudomonas aeruginosa infections. Antimicrobial Agents and Chemotherapy. $2017 ; 61$ (3) (no pagination) (e02136).

38. Dinh A, Wyplosz B, Kerneis S, Lebeaux D, Bouchand F, Duran C, et al. Use of ceftolozane/tazobactam as salvage therapy for infections due to extensively drug-resistant Pseudomonas aeruginosa. International Journal of Antimicrobial Agents. 2017;49(6):782-3.

39. Haidar G, Philips NJ, Shields RK, Snyder D, Cheng S, Potoski BA, et al. Ceftolozane-Tazobactam for the Treatment of Multidrug-Resistant Pseudomonas aeruginosa Infections: Clinical Effectiveness and Evolution of Resistance. Clinical Infectious Diseases. 2017;65(1):11020.

40. Munita JM, Aitken SL, Miller WR, Perez F, Rosa R, Shimose LA, et al. Multicenter Evaluation of Ceftolozane/Tazobactam for Serious Infections Caused by Carbapenem-Resistant Pseudomonas aeruginosa. Clinical Infectious Diseases. 2017;65(1):158-61.

41. Sacha GL, Neuner EA, Athans V, Bass SN, Pallotta A, Rivard KR, et al. Retrospective Evaluation of the Use of Ceftolozane/Tazobactam at a Large Academic Medical Center. Infectious Diseases in Clinical Practice. 2017;25(6):305-9.

42. Xipell M, Bodro M, Marco F, Martinez JA, Soriano A. Successful treatment of three severe MDR or XDR Pseudomonas aeruginosa infections with ceftolozane/tazobactam. Future Microbiology. 2017;12(14):1323-6.

43. Gelfand MS, Cleveland KO. Ceftolozane/tazobactam therapy of respiratory infections due to multidrug-resistant pseudomonas aeruginosa. Clinical Infectious Diseases. 2015;61(5):853-5.

44. Mahmoud A, Shah A, Nutley K, Nicolau DP, Sutherland C, Jain M, et al. Clinical pharmacokinetics of ceftolozane and tazobactam in an obese patient receiving continuous venovenous haemodiafiltration: A patient case and literature review. Journal of Global Antimicrobial

Page 22/28 
Resistance. 2020;21:83-5.

45. Romano MT, Premraj S, Bray JM, Murillo LC. Ceftolozane/tazobactam for pulmonary exacerbation in a 63-year-old cystic fibrosis patient with renal insufficiency and an elevated MIC to Pseudomonas aeruginosa. IDCases. 2020;21 (no pagination).

46. Maddocks S, Fabijan AP, Ho J, Lin RCY, Ben Zakour NL, Dugan C, et al. Bacteriophage therapy of ventilator-associated pneumonia and empyema caused by pseudomonas aeruginosa. American Journal of Respiratory and Critical Care Medicine. 2019;200(9):1179-81.

47. Aguilar G, Ferriols R, Martinez-Castro S, Ezquer C, Pastor E, Carbonell JA, et al. Optimizing ceftolozane-tazobactam dosage in critically ill patients during continuous venovenous hemodiafiltration. Critical Care. 2019;23 (1) (no pagination)(145).

48. Arena F, De Angelis LH, Maglioni E, Contorni M, Cassetta MI, Novelli A, et al. Ceftolozane-tazobactam pharmacokinetics during extracorporeal membrane oxygenation in a lung transplant recipient. Antimicrobial Agents and Chemotherapy. 2019;63 (3) (no pagination)(e02131-18).

49. Carbonell N, Aguilar G, Ferriols R, Huerta R, Ferreres J, Calabuig M, et al. Ceftolozane Pharmacokinetics in a Septic Critically III Patient under Different Extracorporeal Replacement Therapies. Antimicrobial agents and chemotherapy. 2019;28.

50. Davis SE, Ham J, Hucks J, Gould A, Foster R, Ann Justo J, et al. Use of continuous infusion ceftolozane-tazobactam with therapeutic drug monitoring in a patient with cystic fibrosis. American Journal of Health-System Pharmacy. 2019;76(8):501-4.

51. Gonzales Zamora JA, Varadarajalu Y. Fatal Curvularia brain abscess in a heart and kidney transplant recipient. IDCases. $2019 ; 17$ (no pagination)(e00576).

52. Pezzi M, Scozzafava AM, Giglio AM, Vozzo R, Casella PD, Tiburzi SP, et al. Use of Ceftolozane/Tazobactam in a Case of Septic Shock by Puerperal Sepsis. Case Rep Obstet Gynecol. 2019;2019:8463693.

53. Saraca LM, Di Giuli C, Sicari F, Priante G, Lavagna F, Francisci D. Use of Ceftolozane-Tazobactam in Patient with Severe Medium Chronic Purulent Otitis by XDR Pseudomonas aeruginosa. Case rep. 2019;2019:2683701.

54. Alessa MA, Almangour TA, Alhossan A, Alkholief MA, Alhokail M, Tabb DE. Ceftolozane-tazobactam for the treatment of multidrugresistant Pseudomonas aeruginosa pneumonia in a patient receiving intermittent hemodialysis. American Journal of Health-System Pharmacy. 2018;75(9):e184-e8.

55. Frattari A, Savini V, Polilli E, Cibelli D, Talamazzi S, Bosco D, et al. Ceftolozane-tazobactam and Fosfomycin for rescue treatment of otogenous meningitis caused by XDR Pseudomonas aeruginosa: Case report and review of the literature. IDCases. 2018;14 (no pagination)(e00451).

56. Hassan S, Kahn MD, Saraiya N, Nori P. Treatment of a complex orthopaedic infection due to extensively drug-resistant Pseudomonas aeruginosa. BMJ case reports. 2018(pagination).

57. Lewis PO, Cluck DB, Tharp JL, Krolikowski MA, Patel PD. Failure of ceftolozane-tazobactam salvage therapy in complicated pneumonia with lung abscess. Clinical Case Reports. 2018;6(7):1308-12.

58. Monterrubio-Villar J, Rodriguez-Garrido S, Jimenez-Delgado JD. Postoperative soft-tissue infection due to multidrug-resistant Pseudomonas aeruginosa: Usefulness of ceftolozane-tazobactam. Revista Espanola de Quimioterapia. 2018;31(4):374-5.

59. So W, Shurko J, Galega R, Quilitz R, Greene JN, Lee GC. Mechanisms of high-level ceftolozane/tazobactam resistance in Pseudomonas aeruginosa from a severely neutropenic patient and treatment success from synergy with tobramycin. Journal of Antimicrobial Chemotherapy. 2019;74(1):269-71.

60. Stewart A, Roberts JA, Wallis SC, Allworth AM, Legg A, McCarthy KL. Evidence of clinical response and stability of Ceftolozane/Tazobactam used to treat a carbapenem-resistant Pseudomonas Aeruginosa lung abscess on an outpatient antimicrobial program. International Journal of Antimicrobial Agents. 2018;51(6):941-2.

61. Stokem K, Zuckerman JB, Nicolau DP, Wungwattana M, Sears EH. Use of ceftolozane-tazobactam in a cystic fibrosis patient with multidrug-resistant pseudomonas infection and renal insufficiency. Respiratory Medicine Case Reports. 2018;23:8-9.

62. Teleb M, Soto-Ruiz E, Dominguez DC, Antony S. ESBL E coli and P. aeruginosa resistance to ceftolozane-tazobactam in a patient with a liver abscess. the search for an omnipotent antibiotic goes on! Infectious Disorders - Drug Targets. 2018;18(1):81-5.

63. Aye C, Williams M, Horvath R. Multidrug Resistant Pseudomonas Mycotic Pseudoaneurysm following Cardiac Transplant Bridged by Ventricular Assistant Device. Case rep. 2017;2017:1402320.

64. Castaldo N, Givone F, Peghin M, Righi E, Sartor A, Bassetti M. Multidrug-resistant Pseudomonas aeruginosa skin and soft-tissue infection successfully treated with ceftolozane/tazobactam. Journal of Global Antimicrobial Resistance. 2017;9:100-2.

65. Dinh A, Davido B, Calin R, Paquereau J, Duran C, Bouchand F, et al. Ceftolozane/tazobactam for febrile UTI due to multidrug-resistant Pseudomonas aeruginosa in a patient with neurogenic bladder. Spinal Cord Ser Cases. 2017;3:17019.

66. Dominguez AS, Perez-Rodriguez MT, Nodar A, Martinez-Lamas L, Perez-Landeiro A, Casal MC. Successful treatment of MDR Pseudomonas aeruginosa skin and soft-tissue infection with ceftolozane/tazobactam. Journal of Antimicrobial Chemotherapy.

Page 23/28 
2017;72(4):1262-3.

67. Gentile I, Buonomo AR, Maraolo AE, Scotto R, De Zottis F, Di Renzo G, et al. Successful treatment of post-surgical osteomyelitis caused by XDR pseudomonas aeruginosa with ceftolozane/tazobactam monotherapy. Journal of Antimicrobial Chemotherapy.

2017;72(9):2678-9.

68. Hernandez-Tejedor A, Merino-Vega CD, Martin-Vivas A, Ruiz de Luna-Gonzalez R, Delgado-Iribarren A, Gaban-Diez A, et al. Successful treatment of multidrug-resistant Pseudomonas aeruginosa breakthrough bacteremia with ceftolozane/tazobactam. Infection.

2017;45(1):115-7.

69. Jones BM, Smith B, Bland CM. Use of Continuous-Infusion Ceftolozane/Tazobactam in a Multidrug-Resistant Pseudomonas aeruginosa Urinary Tract Infection in the Outpatient Setting. Annals of Pharmacotherapy. 2017;51(8):715-6.

70. Kurtzhalts KE, Mergenhagen KA, Manohar A, Berenson CS. Successful treatment of multidrug-resistant Pseudomonas aeruginosa pubic symphysis osteomyelitis with ceftolozane/tazobactam. BMJ Case Reports. 2017;2017 (no pagination)(bcr-2016-217005).

71. MacVane SH, Pandey R, Steed LL, Kreiswirth BN, Chen L. Emergence of ceftolozane-tazobactam-resistant Pseudomonas aeruginosa during treatment is mediated by a single AmpC structural mutation. Antimicrobial Agents and Chemotherapy. $2017 ; 61$ (12) (no pagination)(e01183).

72. Peghin M, Maiani M, Castaldo N, Givone F, Righi E, Lechiancole A, et al. Ceftolozane/tazobactam for the treatment of MDR Pseudomonas aeruginosa left ventricular assist device infection as a bridge to heart transplant. Infection. 2018;46(2):263-5.

73. Schwarz ER, Oikonomou KG, Reynolds M, Kim J, Balmiki RL, Sterling SA. Extranodal NK/T-cell lymphoma, nasal type, presenting as refractory pseudomonas aeruginosa facial cellulitis. Journal of Investigative Medicine High Impact Case Reports. 2017;5(3).

74. Jolliff JC, Ho J, Joson J, Heidari A, Johnson R. Treatment of Polymicrobial Osteomyelitis with Ceftolozane-Tazobactam: Case Report and Sensitivity Testing of Isolates. Case rep. 2016;2016:1628932.

75. Kuti JL, Ghazi IM, Quintiliani R, Shore E, Nicolau DP. Treatment of multidrug-resistant Pseudomonas aeruginosa with ceftolozane/tazobactam in a critically ill patient receiving continuous venovenous haemodiafiltration. International Journal of Antimicrobial Agents. 2016;48(3):342-8.

76. Patel UC, Nicolau DP, Sabzwari RK. Successful Treatment of Multi-Drug Resistant Pseudomonas aeruginosa Bacteremia with the Recommended Renally Adjusted Ceftolozane/Tazobactam Regimen. Infectious Diseases and Therapy. 2016;5(1):73-9.

77. Vickery SB, McClain D, Wargo KA. Successful Use of Ceftolozane-Tazobactam to Treat a Pulmonary Exacerbation of Cystic Fibrosis Caused by Multidrug-Resistant Pseudomonas aeruginosa. Pharmacotherapy. 2016;36(10):e154-e9.

78. Soliman R, Lynch S, Meader E, Pike R, Turton JF, Hill RL, et al. Successful ceftolozane/tazobactam treatment of chronic pulmonary infection with pan-resistant Pseudomonas aeruginosa. JMM Case Reports. 2015;2(2):e000025.

79. Gudiol, Albasanz, Fernandez-Cruz, Puerta, Hakki, Ruiz, et al., editors. Ceftolozane-tazobactam for the treatment of bloodstream infection due to Pseudomonas aeruginosa in neutropenic cancer patients: a real-life experience (ZENITH study). ECCMID; 2020.

80. Caffrey, Piehl, Lopes, Puzniak, Laplante, editors. Comparative effectiveness of ceftolozane/tazobactam versus aminoglycosides or polymyxins in multidrug resistant Pseudomonas aeruginosa infections. ECCMID; 2020.

81. Trisler, Tamma, Avdic, editors. Comparison of Outcomes Between Patients with and without Cystic Fibrosis Treated with CeftolozaneTazobactam for Pseudomonas aeruginosa Infections. IDWeek; 2019.

82. Sheffield, Nelson, O'Neal, Gould, Bouchard, Nicolau, et al., editors. The use of continuous infusion Ceftolozane/tazobactam for resistant gram-negative bacterial infections: a case series. ACCP; 2019.

83. Mills, MacWhinnie, Do, editors. Evaluating the Impact of Ceftolozane/Tazobactam on Clinical Outcomes in Patients with Multi-Drugresistant Pseudomonas aeruginosa Pneumonia. IDWeek; 2019.

84. Hart, Gallagher, et al., editors. Ceftolozane-tazobactam (C/T) Treatment Outcomes in Immunocompromised (IC) Patients with Multidrug-Resistant (MDR) Pseudomonas aeruginosa (PA) Infections. IDWeek; 2019.

85. Cabrera, Tran, Miller, Dinh, Hanson, Munita, et al., editors. Clinical and Microbiological Outcomes Associated with Real-World Use of Ceftolozane/Tazobactam. IDWeek; 2019.

86. Tordato, Casana, Lagioia, Morelli, editors. Efficacy and safety of ceftolozane/tazobactam as salvage therapy in severely ill patients. ECCMID; 2018.

87. Puzniak, Rao, Gundrum, et al., editors. Real world evaluation of patient characteristics and outcomes of patients treated with ceftolozane/tazobactam across 253 US hospitals. IDWeek; 2018.

88. Puzniak, Rao, Gundrum, editors. Real world evaluation of ceftolozane/tazobactam treatment for Pseudomonas across 253 US hospitals. SCCM; 2018. 
89. Pogue, Sanagaram, Merchant, Raina, Puzniak, editors. Real world clinical experience with ceftolozane/tazobactam (C/T) for the treatment of complicated urinary tract infections (CUTI) and complicated intraabdominal infections (clAl) due to Pseudomonas aeruginosa (PSA): an electronic medical record database review in the United States. ECCMID; 2018.

90. Jorgensen, Trinh, Lagnf, Bhatia, Estrada, Simon, et al., editors. Multicentre evaluation of ceftolozane-tazobactam for multidrug-resistant Pseudomonas aeruginosa infections. ECCMID; 2018.

91. Jorgensen, et al., editors. Multicenter evaluation of $\mathrm{C} / \mathrm{T}$ monotherapy vs. combination therapy for MDR P. aeruginosa. ASM Microbe; 2018.

92. Jayakumar, Leuthner, Kullar, Hewlett, Nguyen, Puzniak, editors. Real-world evaluation of ceftolozane/tazobactam (C/T) in severely ill patients with sepsis and/or bacteraemia. ECCMID; 2018.

93. Hooper, Elsayed, Bombassaro, Bailey, Bondy, editors. Successful Treatment of Chronic Spinal Osteomyelitis Caused by Multidrug Resistant Pseudomonas aeruginosa with Ceftolozane-Tazobactam and Surgical Intervention. CANADIAN JOURNAL OF HOSPITAL PHARMACY; 2018.

94. Hirsch, Hart, Piche, Cubillos, Beaulac, Bandali, et al., editors. A Multi-Center Evaluation of Outcomes following Treatment with Ceftolozane-Tazobactam2018.

95. Henry, Schoen, Puzniak, Raddatz, Van Schooneveld, Bergman, editors. Ceftolozane-Tazobactam Use and Outcomes at an Academic Transplant Center. ACCP; 2018.

96. Gioia, Sánchez, Martín-Dávila, Pintado, Reilly, Ruiz-Garbajosa, et al., editors. Ceftolozane-tazobactam for the treatment of Pseudomonas aeruginosa infection in a tertiary hospital: clinical outcome and develop of resistance. ECCMID; 2018.

97. Elabor, Molnar, King, Gallagher, editors. Ceftolozane/tazobactam for the Treatment of Multidrug Resistant Pseudomonas aeruginosa Infections in Immunocompromised Patients: A Multi Center Study. IDWeek; 2018.

98. Leuthner, Kullar, Jayakumar, Hewlett, Nguyen, Puzniak, editors. Real-world Evaluation of Ceftolozane/Tazobactam (C/T) Use and Clinical Outcomes at an Academic Medical Center in Las Vegas. IDWeek; 2017.

99. Nathan, Alvarado, Prokesch, Quyen, Sleweon, Schroeder, et al., editors. Ceftolozane/Tazobactam: Outpatient Treatment of GramNegative Infections at Physician Office Infusion Centers (POICs). IDWeek; 2016.

100. Iovleva, Marshall, Perez, Ray, Jacobs, Bonomo, editors. Ceftazidime/avibactam and ceftolozane/tazobactam in treatment of pulmonary infections by Imipenem resistant Pseudomonas aeruginosa. IDWeek; 2016.

101. Merck \& Co. Data on file: ASPECT-cUTI Clinical Study Report. 2014.

102. Merck \& Co. Data on file: ASPECT-clAI Clinical Study Report. 2014.

\section{Figures}




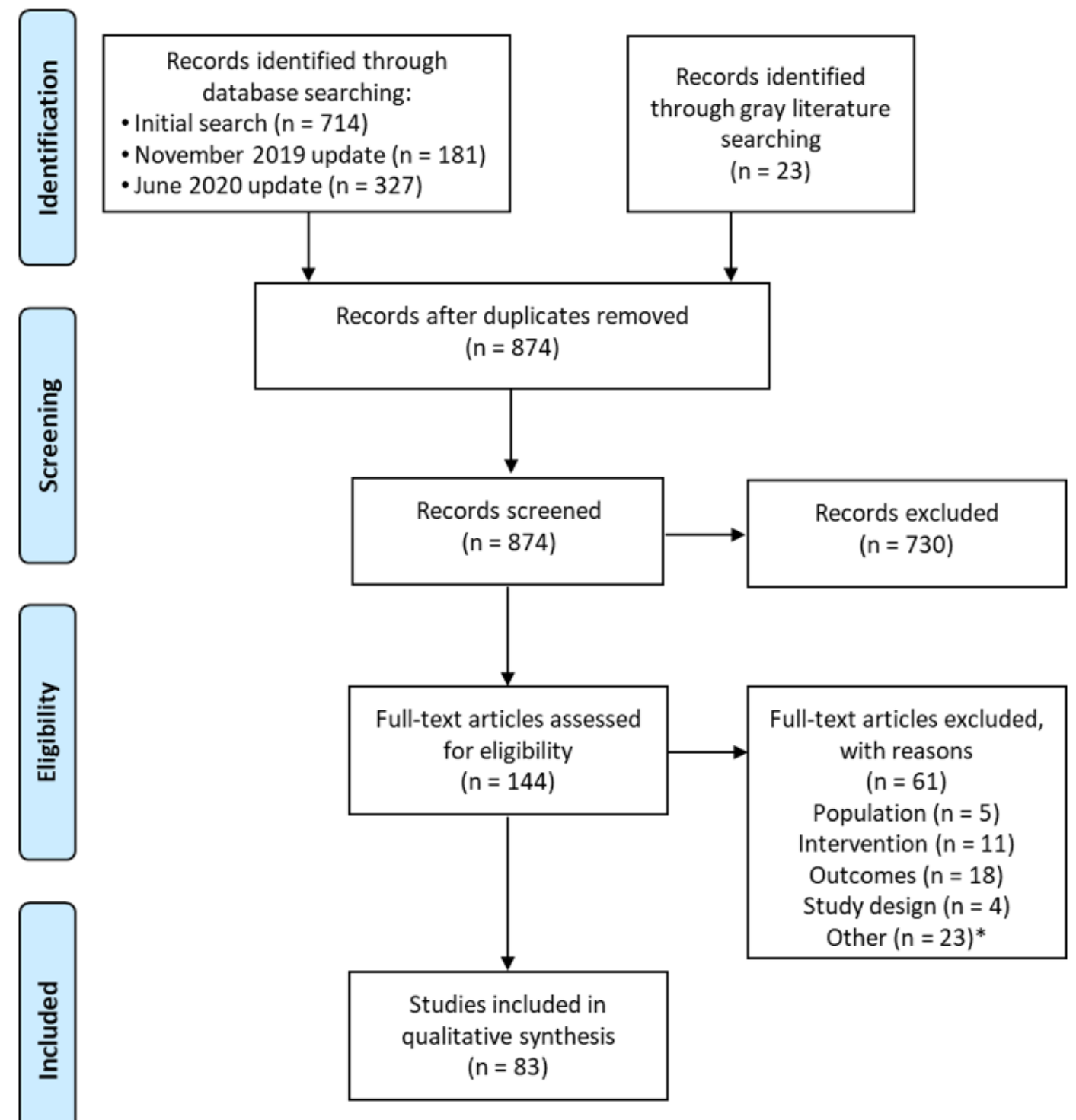

Figure 1

PRISMA diagram. *Other includes duplicates identified at the full-text stage and records that were identified as either conference proceedings or pre-publication manuscripts in the initial or November 2019 search and then identified again as full-text publications in the later update. 


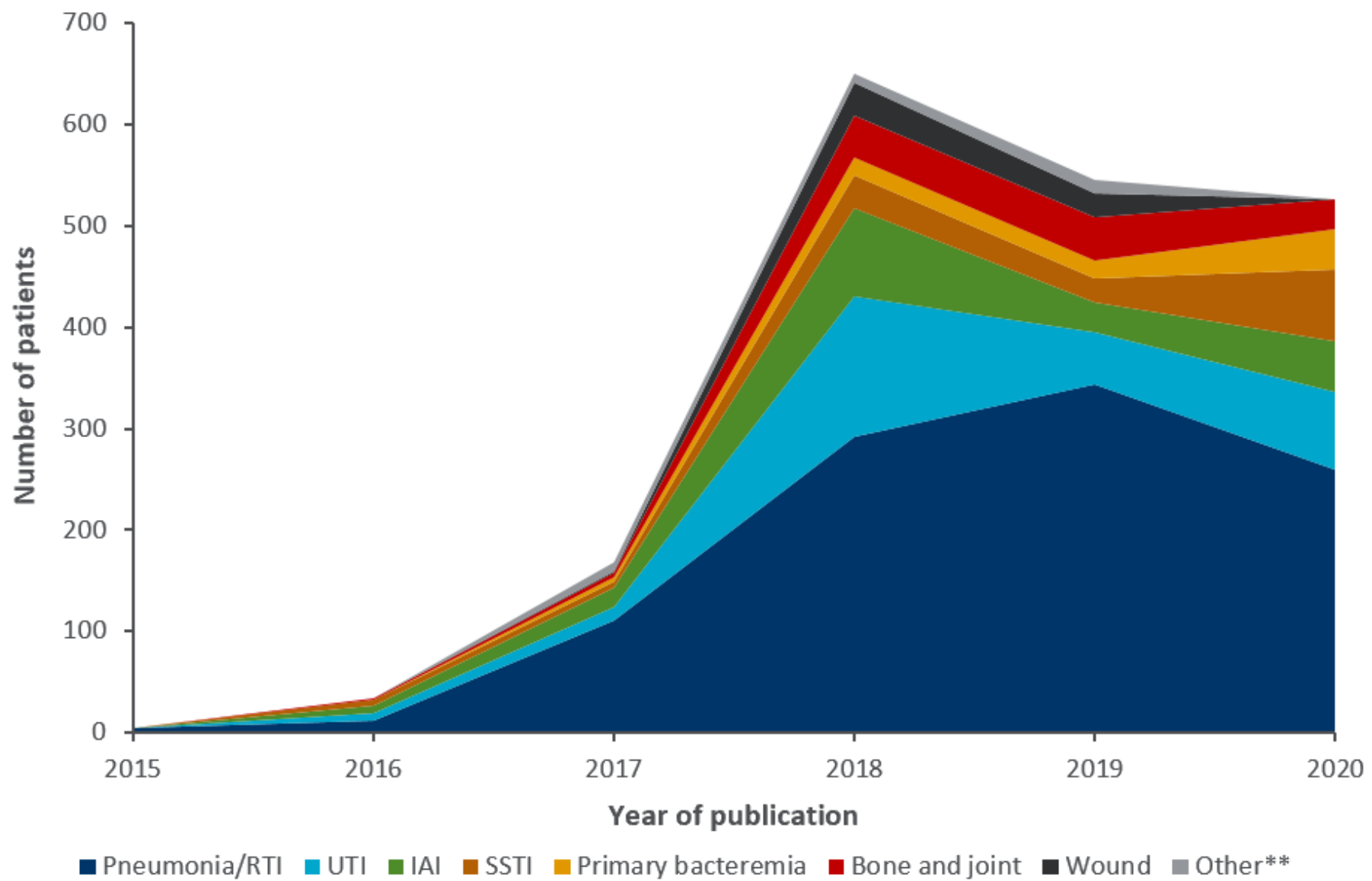

Figure 2

Infections of patients treated with $\mathrm{C} / \mathrm{T}$ by publication year* *xcluding patients for which infection was not specified. **0ther includes genital infection, CNS infection, liver abscess, mediastinitis, device-related infections, vascular infection, and otitis and mastoiditis. CNS: Central nervous system; C/T: Ceftolozane/tazobactam; IAI: Intra-abdominal infection; RTI: Respiratory tract infection; SSTI: Skin and soft tissue infection; UTI: Urinary tract infection.

A. $P$. aeruginosa resistance profile, 2015-2017 studies ( $\mathrm{N}=27$ studies; $\mathrm{n}=195$ patients)

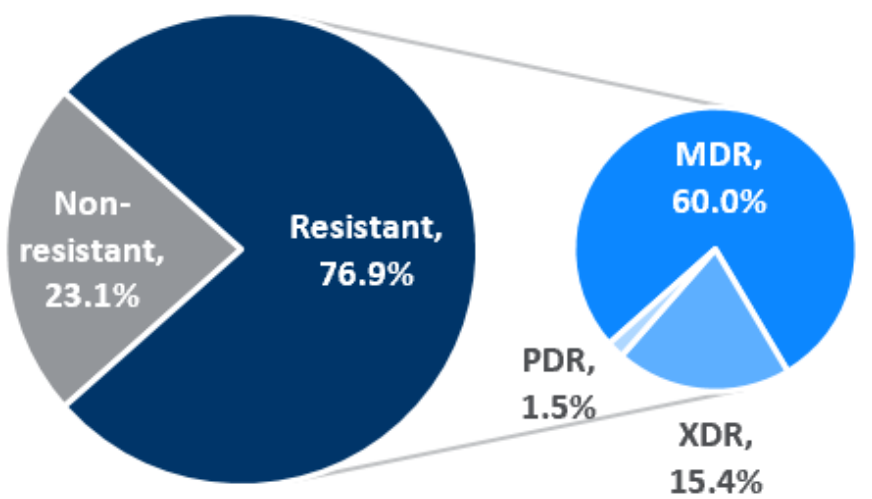

B. P. aeruginosa resistance profile, 2018-2020 studies ( $N=56$ studies; $n=1,989$ patients)

\section{Figure 3}

P. aeruginosa resistance profile in studies identified in 2015-2017 and 2018-2020 MDR: Multidrug-resistant; PDR: Pandrug-resistant; XDR: Extensively-drug-resistant. 


\section{Supplementary Files}

This is a list of supplementary files associated with this preprint. Click to download.

- Supplementarymaterial.docx 